\title{
Performance of DS-CDMA Systems With Optimal Hard-Decision Parallel Interference Cancellation
}

\author{
Remco van der Hofstad and Marten J. Klok
}

\begin{abstract}
We study a multiuser detection system for code-division multiple access (CDMA). We show that applying multistage hard-decision parallel interference cancellation (HD-PIC) significantly improves performance compared to the matched filter system. In (multistage) HD-PIC, estimates of the interfering signals are used iteratively to improve knowledge of the desired signal. We use large deviation theory to show that the bit-error probability (BEP) is exponentially small when the number of users is fixed and the processing gain increases. We investigate the exponential rate of the BEP after several stages of HD-PIC. We propose to use the exponential rate of the BEP as a measure of performance, rather than the signal-to-noise ratio (SNR), which is often not reliable in multiuser detection models when the system is lightly loaded.

We show that the exponential rate of the BEP remains fixed after a finite number of stages, resulting in an optimal hard-decision system. When the number of users becomes large, the exponential rate of the BEP converges to $(\log 2) / 2-1 / 4$. We provide guidelines for the number of stages necessary to obtain this asymptotic exponential rate. We also give Chernoff bounds on the BEPs. These estimates show that the BEPs are quite small as long as $k=o(n / \log n)$ when the number of stages of HD-PIC is fixed, and even exponentially small when $k=\mathcal{O}(n)$ for the optimal HD-PIC system, and where $k$ is the number of users in the system and $n$ is the processing gain. Finally, we extend the results to the case where the number of stages depends on $k$ in a certain manner. The above results are proved for a noiseless channel, and we argue that we expect similar results in a noisy channel as long as the two-sided spectrum of the noise decreases proportionally to $n$.
\end{abstract}

Index Terms-Code-division multiple access (CDMA), exponential rate, hard-decision parallel interference cancellation (HD-PIC), large deviations, optimal hard-decision system, performance measures.

\section{INTRODUCTION}

C URRENTLY, the third-generation (3G) mobile communication system is being introduced. This system is based on code-division multiple access (CDMA). The performance

Manuscript received January 22, 2002; revised May 30, 2003. The work of M. J. Klok was supported in part under a grant from The Netherlands Organization for Scientific Research (NWO), and in part under a grant of the Stieltjes Institute for Mathematics. The work of R. van der Hofstad was supported in part by the University of British Columbia during a visit during October-December 2001.

R. van der Hofstad was with the Faculty of Information Technology and Systems, Delft University of Technology, 2628 CD Delft, The Netherlands. $\mathrm{He}$ is now with the Department of Mathematics, Eindhoven University of Technology, P. O. Box 513, 5600 MB Eindhoven, The Netherlands (e-mail: rhofstad@win.tue.nl)

M. J. Klok is with the Faculty of Information Technology and Systems, Delft University of Technology, 2628 CD Delft, The Netherlands (e-mail: m.j.klok@its.tudelft.nl).

Communicated by V. Veeravalli, Associate Editor for Detection and Estimation.

Digital Object Identifier 10.1109/TIT.2003.818413 of CDMA is mainly limited by interference from other users: the multiple-access interference. Particularly, the susceptibility to the near-far situation, which can significantly reduce capacity in the absence of good power control, is a problem. Therefore, there exists great interest in techniques which improve the capacity of CDMA receivers (see [17], [2], [18], and references therein). Initial research on multiuser receivers for CDMA has demonstrated the potential improvements in capacity and near-far resistance. The best known technique is a maximum-likelihood sequence estimator [21], which obtains jointly optimum decisions for all users. A straightforward technique is called interference cancellation, see [18], [16, Ch. 4], [11], [20], and references therein. Interference cancellation, especially hard-decision parallel interference cancellation (HD-PIC), is a promising and practical technique for uplink receivers (see [18]).

In the literature, not much attention has been given to rigorous analytical results for (HD-)PIC systems. The papers that exist on this subject focus on approximating the signal-to-noise ratio (SNR), for example [1] and [22]. However, using the SNR as a measure of performance implicitly relies on Gaussian assumptions. For the PIC system this implicit assumption is false when the system is lightly loaded, i.e., when the number of users is small compared to the processing gain (see Section II-F).

We use large deviation theory [5], [10] to obtain analytical results for the bit-error probability (BEP). More precisely, rather than calculating the SNR, we calculate the exponential rate of the BEP. We will first explain the significance of the exponential rate. Suppose $p_{n}$ is a sequence of probabilities and $p_{n} \rightarrow 0$ as $n \rightarrow \infty$. In our setting, $n$ stands for the processing gain. Often, this decay is exponentially in $n$, so that we investigate the exponential rate defined as

$$
I=-\lim _{n \rightarrow \infty} \frac{1}{n} \log p_{n}
$$

For finite, but not too small $n$, we can read this as

$$
p_{n} \sim e^{-n I} .
$$

Thus, the probability $p_{n}$ is mainly characterized by its exponential rate.

It turns out that the BEP for matched filter (MF) and HD-PIC systems are exponentially small when the system is lightly loaded. This makes possible the use of the exponential rate as a measure for the performance in order to compare different systems.

For the MF model, the SNR and the exponential rate are asymptotically equivalent, meaning that when the number of users is sufficiently large, both substitution of the SNR into the Gaussian error function and the use of the exponential rate imply that the BEP has the same asymptotic form. This is shown in 
Section II-F. In [19], an MF model is investigated using large deviation techniques. However, the emphasis there lies more on sampling techniques. For a one-stage soft-decision PIC model, results have been obtained in [8]. In that paper, large deviation techniques are used to prove properties of the exponential rate and the BEP. In [9] and [13], various results have been proven for the HD-PIC system.

In this paper, we start by repeating results of the single-stage and multistage HD-PIC of [9] and [13]. We extend these results to values of the processing gain and the number of users that are comparable using Chernoff bounds.

The main novelty of this paper is that we obtain results for a system with infinite stages of HD-PIC, the so-called optimal $H D$-PIC system. One of the main results is that after finitely many iterations, the exponential rate does not increase any further. In other words, applying more stages of interference cancellation no longer improves performance. We will give a theoretic upper bound on the number of stages required, and a heuristic explanation of how large we believe this number to be for many users.

A striking result is that the obtained exponential rate is strictly positive, regardless of the number of users. We will give bounds on this exponential rate, and subsequently derive bounds on the BEP. Furthermore, we give intuition on the number of stages necessary to obtain this exponential rate. We will also extend the results of the optimal HD-PIC system to the case where the number of stages of HD-PIC depends on the number of users in a certain manner.

\section{SYSTEM AND BACKGROUND}

In this section, we explain the system, and give previous results concerning HD-PIC.

\section{A. System Model}

We first introduce a mathematical model for CDMA systems. We define the data signal $b_{m}(t)$ of the $m$ th user as $b_{m}(t)=$ $b_{m\lfloor t / T\rfloor}$, for $1 \leq m \leq k$, where

$$
b_{m}=\left(\ldots, b_{m,-1}, b_{m 0}, b_{m 1}, \ldots\right) \in\{-1,+1\}^{\mathbb{Z}}
$$

and where, for $x \in \mathbb{R},\lfloor x\rfloor$ denotes the largest integer smaller than or equal to $x$. For each user $m, 1 \leq m \leq k$, we have a coding sequence

$$
a_{m}=\left(\ldots, a_{m,-1}, a_{m 0}, a_{m 1}, \ldots\right) \in\{-1,+1\}^{\mathbb{Z}}
$$

and we put $a_{m}(t)$, the rectangular spreading signal, generated by $\left(a_{m}\right)_{m=-\infty}^{\infty}$, i.e., $a_{m}(t)=a_{m\left\lfloor t / T_{c}\right\rfloor}$, where $T_{c}=T / n$, for some integer $n$. The variable $n$ is often called the processing gain and is equal to the length of the coding sequences of all users in the system.

The transmitted coded signal of the $m$ th user is then

$$
s_{m}(t)=\left(2 P_{m}\right)^{1 / 2} b_{m}(t) a_{m}(t) \cos \left(\omega_{c} t\right), \quad 1 \leq m \leq k
$$

where $P_{m}$ is the power of the $m$ th user and $\omega_{c}$ the carrier frequency. The total transmitted signal is given by

$$
r(t)=\sum_{j=1}^{k} s_{j}(t)
$$

In practice, the signals do not need to be synchronized, i.e., it is not necessary for all users to transmit using the same time grid. However, for technical reasons, we make this assumption In [19], an asynchronous MF system is investigated, using large deviations techniques. One important result in that paper is that the asynchronous system has many similarities compared to the synchronous system. In fact, the exponential rate for the synchronous and asynchronous system are the same. For the more advanced HD-PIC system, this idea carries over, so that the results investigated in this paper turn out not to differ from those for an asynchronous system. Indeed, we expect that the asynchronous model has the same exponential rate of the BEP as the synchronized model. Therefore, the synchronicity assumption is without loss of generality.

We assume that there is no additive white Gaussian noise (AWGN). Thus, we are dealing with a perfect channel. However, we believe that many of our results remain true even when there is little AWGN. For the details of the assumptions on the AWGN, in particular what it means to have little AWGN, see Section II-D.

To retrieve the data bit $b_{m 0}$, the signal $r(t)$ is multiplied by $a_{m}(t) \cos \omega_{c} t$ and then averaged over $[0, T]$. For simplicity, we pick $\omega_{c} T_{c}=\pi f_{c}$, where $f_{c} \in \mathbb{N}$ to get (cf. [8, eqs. (3) and (4)])

$$
\begin{aligned}
& \frac{1}{T} \int_{0}^{T} r(t) a_{m}(t) \cos \left(\omega_{c} t\right) d t \\
& \quad=\left(\frac{P_{m}}{2}\right)^{1 / 2} b_{m 0}+\sum_{\substack{j=1 \\
j \neq m}}^{k}\left(\frac{P_{j}}{2}\right)^{1 / 2} b_{j 0} \frac{1}{n} \sum_{i=0}^{n-1} a_{j i} a_{m i}
\end{aligned}
$$

where we recall that $n$ is the length of the coding sequences and $k$ is the number of users in the system.

As is seen from (4), the decoded signal consists of the desired bit and interference due to the other users. In the ideal situation the vectors $\left(a_{m 0}, \ldots, a_{m, n-1}\right)$ and $\left(a_{j 0}, \ldots, a_{j, n-1}\right), j \neq m$, would be orthogonal, so that $\sum_{i=1}^{n} a_{j i} a_{m i}=0$. In practice, the $a$-sequences are generated by a random number generator. To model the pseudorandom sequence $a$, let $A_{m i}, m=1,2, \ldots, k$, $i=1,2, \ldots, n$, be an array of independent and identically distributed (i.i.d.) random variables with distribution

$$
\mathbb{P}\left(A_{11}=+1\right)=\mathbb{P}\left(A_{11}=-1\right)=1 / 2 .
$$

Assuming the coding sequences to be random, we model the signal of (4) as

$$
\left(\frac{P_{m}}{2}\right)^{1 / 2} b_{m 0}+\sum_{\substack{j=1 \\ j \neq m}}^{k}\left(\frac{P_{j}}{2}\right)^{1 / 2} b_{j 0} \frac{1}{n} \sum_{i=1}^{n} A_{j i} A_{m i}
$$

where we have replaced $i=0, \ldots, n-1$ by $i=1, \ldots, n$, for notational convenience.

In practice, the $a$-sequences are not chosen as i.i.d. sequences. Rather, they are carefully chosen to have good correlation properties. Examples are Gold sequences [6] or Kasami sequences [12]. However, it is common in the literature to use random sequences, so that a detailed analysis is possible. Better performance can be achieved for well-chosen deterministic sequences.

We let $\hat{b}_{m 0}^{(1)}$ be the estimator for $b_{m 0}$ given by

$$
\operatorname{sgnr}_{m}\left\{\left(\frac{P_{m}}{2}\right)^{1 / 2} b_{m 0}+\sum_{\substack{j=1 \\ j \neq m}}^{k}\left(\frac{P_{j}}{2}\right)^{1 / 2} b_{j 0} \frac{1}{n} \sum_{i=1}^{n} A_{j i} A_{m i}\right\},
$$


where, for $x \in \mathbb{R}$, the randomized sign-function is defined as

$$
\operatorname{sgnr}_{m}(x)= \begin{cases}+1, & x>0 \\ U_{m}, & x=0 \\ -1, & x<0\end{cases}
$$

with $\mathbb{P}\left(U_{m}=-1\right)=\mathbb{P}\left(U_{m}=+1\right)=1 / 2$. The random variables $U_{m}$ are independent from all other random variables in the system. Note that the $m$ th user always makes the same decision when its signal produces a zero. There are other ways to define the sign-function, such as the choice where every time a zero is detected, a new independent random $U$ is chosen. Another option is to let the sign of 0 be equal to 0 . We choose the definition in (6) for technical reasons only. We will comment more on other choices of sign functions in Section III. We use the notation ${ }^{(1)}$ in $\hat{b}_{m 0}^{(1)}$ to indicate that this is a tentative decision only.

We now describe the hard-decision procedure. The powers $P_{m}$ are assumed to be known. We estimate the data signal $s_{j}(t)$ for $t \in[0, T]$ by (recall (2))

$$
\hat{s}_{j}^{(1)}(t)=\left(2 P_{j}\right)^{1 / 2} \hat{b}_{j 0}^{(1)} a_{j}(t) \cos \left(\omega_{c} t\right) .
$$

We next estimate the total interference for the $m$ th user in $r(t)$ due to the other users by (recall (3))

$$
\hat{r}_{m}^{(1)}(t)=\sum_{\substack{j=1 \\ j \neq m}}^{k} \hat{s}_{j}^{(1)}(t)
$$

We use the above to find a better estimate of the data bit $b_{m 0}$, denoted by $\hat{b}_{m 0}^{(2)}$, which is the $\operatorname{sgnr}_{m}$ of

$$
\begin{aligned}
\frac{1}{T} \int_{0}^{T}\left(r(t)-\hat{r}_{m}^{(1)}(t)\right) a_{m}(t) \cos \left(\omega_{c} t\right) d t \\
=\left(\frac{P_{m}}{2}\right)^{1 / 2} b_{m 0} \\
\quad+\sum_{\substack{j=1 \\
j \neq m}}^{k}\left(\frac{1}{n} \sum_{i=1}^{n} A_{j i} A_{m i}\right)\left(\frac{P_{j}}{2}\right)^{1 / 2}\left(b_{j 0}-\hat{b}_{j 0}^{(1)}\right) .
\end{aligned}
$$

We are now interested in $\mathbb{P}\left(\hat{b}_{m 0}^{(2)} \neq b_{m 0}\right)$, which is the probability of a bit error after one stage of interference cancellation. In the following, we will see that for the systems under consideration the exponential rate of this probability is indeed larger than the exponential rate of $\mathbb{P}\left(\hat{b}_{m 0}^{(1)} \neq b_{m 0}\right)$, where $\mathbb{P}\left(\hat{b}_{m 0}^{(1)} \neq b_{m 0}\right)$ denotes the probability of a bit error without cancellation. This motivates a repetition of the previous procedure. Similarly to (7), we obtain the estimates $\hat{b}_{m 0}^{(s)}$, which are the $\operatorname{sgnr}_{m}$ of

$$
\left(\frac{P_{m}}{2}\right)^{1 / 2} b_{m 0}+\sum_{\substack{j=1 \\ j \neq m}}^{k}\left(\frac{1}{n} \sum_{i=1}^{n} A_{j i} A_{m i}\right)\left(\frac{P_{j}}{2}\right)^{1 / 2}\left(b_{j 0}-\hat{b}_{j 0}^{(s-1)}\right) .
$$

This is called multistage HD-PIC. When we have applied $s$ steps of interference cancellation we speak of $s$-stage HD-PIC and the corresponding $\mathrm{BEP}$ is $\mathbb{P}\left(\hat{b}_{m 0}^{(s+1)} \neq b_{m 0}\right)$.

\section{B. Reformulation of the Problem}

We can write the probability of a bit-error in a more convenient way. Namely, because $b_{m i}^{2}=1$, we have

$$
\begin{aligned}
& \left(\frac{P_{m}}{2}\right)^{1 / 2} b_{m 0}+\sum_{\substack{j=1 \\
j \neq m}}^{k}\left(\frac{P_{j}}{2}\right)^{1 / 2} b_{j 0} \frac{1}{n} \sum_{i=1}^{n} A_{j i} A_{m i} \\
& =b_{m 0}\left(\left(\frac{P_{m}}{2}\right)^{1 / 2}+\sum_{\substack{j=1 \\
j \neq m}}^{k}\left(\frac{P_{j}}{2}\right)^{1 / 2} \frac{1}{n} \sum_{i=1}^{n} b_{j 0} A_{j i} b_{m 0} A_{m i}\right)
\end{aligned}
$$

Since $A_{j i} \stackrel{d}{=} b_{j 0} A_{j i}$, where $\stackrel{d}{=}$ denotes equality in distribution, we have

$$
\begin{aligned}
& \mathbb{P}\left(\hat{b}_{m 0}^{(1)} \neq b_{m 0}\right) \\
& \quad=\mathbb{P}\left(\frac{\hat{b}_{m 0}^{(1)}}{b_{m 0}} \neq 1\right) \\
& \quad=\mathbb{P}\left(\operatorname{sgnr}_{m}\left(Z_{m}^{(1)}\right) \neq 1\right)=\mathbb{P}\left(Z_{m}^{(1)}<0\right)+\frac{1}{2} \mathbb{P}\left(Z_{m}^{(1)}=0\right)
\end{aligned}
$$

where $Z_{m}^{(1)}$, for $1 \leq m \leq k$, is defined as

$$
Z_{m}^{(1)}=P_{m}^{1 / 2}+\sum_{\substack{j=1 \\ j \neq m}}^{k} P_{j}^{1 / 2} \frac{1}{n} \sum_{i=1}^{n} A_{j i} A_{m i}
$$

We can easily bound

$$
\mathbb{P}\left(Z_{m}^{(1)}<0\right) \leq \mathbb{P}\left(\hat{b}_{m 0}^{(1)} \neq b_{m 0}\right) \leq \mathbb{P}\left(Z_{m}^{(1)} \leq 0\right) .
$$

Note that the above bounds are completely independent of the choice of the sign function in (6). The upper and lower bound in (9) can be shown to be almost equal when $k \geq 3$ and $n$ is large.

Similarly, we define for $s \geq 2$ and $1 \leq m \leq k$

$$
\begin{aligned}
Z_{m}^{(s)}=P_{m}^{1 / 2}+ & \sum_{\substack{j=1 \\
j \neq m}}^{k} P_{j}^{1 / 2}\left(\frac{1}{n} \sum_{i=1}^{n} A_{j i} A_{m i}\right) \\
& \times\left[1-\operatorname{sgnr}_{j}\left(Z_{j}^{(s-1)}\right)\right]
\end{aligned}
$$

to obtain as in (9)

$$
\mathbb{P}\left(Z_{m}^{(s)}<0\right) \leq \mathbb{P}\left(\hat{b}_{m 0}^{(s)} \neq b_{m 0}\right) \leq \mathbb{P}\left(Z_{m}^{(s)} \leq 0\right) .
$$

We will now investigate the performance of HD-PIC. We will use as a measure of performance the exponential rate of a bit error, defined by

$$
H_{k ; m}^{(s)}=-\lim _{n \rightarrow \infty} \frac{1}{n} \log \mathbb{P}\left(\hat{b}_{m}^{(s)} \neq b_{m}\right) .
$$

For systems with equal powers, the users are exchangeable, so that the above quantity does not depend on $m$. In that case, we define $H_{k}^{(s)}=H_{k ; m}^{(s)}$ for all $m$.

We will often use the system with equal powers as a reference model. We will denote this model by the simple system. Without loss of generality, we then take $P_{m}=2$, so that the factors $\left(P_{m} / 2\right)^{1 / 2}$ disappear. 


\section{Previous Results for HD-PIC}

In [8], it is shown that the exponential rate without interference cancellation $H_{k}^{(1)}$ (denoted there by $I_{k}$ ), for the simple system for $k \geq 3$, is given by ${ }^{1}$

$$
H_{k}^{(1)}=\frac{k-2}{2} \log \left(\frac{k-2}{k-1}\right)+\frac{k}{2} \log \left(\frac{k}{k-1}\right) .
$$

In [9], we have obtained an analytic formula for the rate after one stage of HD-PIC.

Theorem II.1: When all powers are equal

$$
H_{k}^{(2)}=\min _{1 \leq r \leq k-1} H_{k, r}^{(2)}
$$

where

$$
H_{k, r}^{(2)}=\sup _{s \leq 0, t \leq 0}\{-\log h(s, t)\}
$$

with $h(s, t)=h_{k, r}(s, t)$ equal to

$$
2^{-r} \sum_{j=-r}^{r}\left(\begin{array}{c}
r \\
\frac{r+j}{2}
\end{array}\right) e^{s+2 s j+t j+t j^{2}}(\cosh t j)^{k-r-1} .
$$

In the statement of the theorem, one should not confuse $H_{k, r}^{(2)}$ with $H_{k ; m}^{(2)}$ in (12).

The proof in [9] shows that the problem of finding the exponential rate of the BEP after one stage of HD-PIC is split into two optimization problems. First, $r$ represents the number of bits that have been estimated wrongly in the first stage. For every $r$, we solve the large deviation minimization problem, leading to $H_{k, r}^{(2)}$. Here, the function $h(s, t)$ is the combined moment-generating function of $\sum_{m=1}^{r} Z_{m}^{(1)}$ and $Z_{1}^{(2)}$, and, as often is the case in large deviation theory, the rate is the Legendre transform of the log of the moment-generating function. Then we minimize over $r$ to obtain the rate $H_{k}^{(2)}$, which corresponds to finding the number of bit errors in the first stage that minimizes the exponential rate of the BEP in the second stage.

We can give an analytical expression for $H_{k, 1}^{(2)}$, similar to the rate for the MF system, that reads

$$
\begin{aligned}
H_{k, 1}^{(2)} & =\frac{3}{4} \log 3-\log 2 \\
& +\frac{2 k-5}{4} \log \left(\frac{2 k-5}{2 k-4}\right)+\frac{2 k-3}{4} \log \left(\frac{2 k-3}{2 k-4}\right) .
\end{aligned}
$$

For general $r$, we cannot obtain a closed-form expression for $H_{k, r}^{(2)}$. However, standard numerical packages allow us to compute $H_{k, r}^{(2)}$ for all $k$ and $r$. In Fig. $1, H_{k, r}^{(2)}$ is shown for $r=$ $1, \ldots, 5$. Observe that the optimal $r$ equals 1 for $k \leq 9$. In probability language this means that, for large $n$, the BEP caused by two or more bit errors in the first stage is negligible compared to the BEP caused by one bit error in the first stage. For $k=$ $10, \ldots, 25, r=2$ will give the minimal rate, meaning that "typically" a bit error in the second stage (after HD-PIC) is caused by two bit errors in the first stage. This is further illustrated in Table I, where the optimal $r_{k}$ is given for $k=3, \ldots, 250$.

${ }^{1} \mathrm{~A}$ different definition of the sign function is used there, but this does not influence the results.

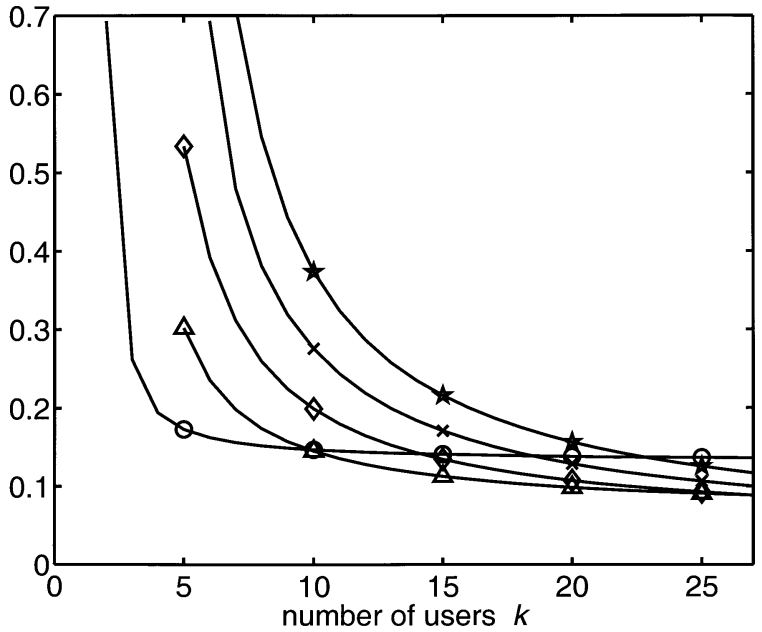

Fig. 1. Exponential rates $H_{k, r}^{(2)}$ for $r=1, \ldots, 5$ indicated with $\circ, \triangle, \diamond, \times, \star$, respectively.

TABLE I

OPTIMAL VALUE $r_{k}$ FOR $k=3, \ldots, 250$

\begin{tabular}{cc}
$k$ & $r_{k}$ \\
\hline$\{3, \ldots, 9\}$ & 1 \\
$\{10, \ldots, 26\}$ & 2 \\
$\{27, \ldots, 51\}$ & 3 \\
$\{52, \ldots, 84\}$ & 4 \\
$\{85, \ldots, 125\}$ & 5 \\
$\{126, \ldots, 174\}$ & 6 \\
$\{175, \ldots, 231\}$ & 7 \\
$\{232, \ldots, 250\}$ & 8 \\
\hline
\end{tabular}

For $1 / k \rightarrow 0$, a Taylor expansion of (13) yields

$$
H_{k}^{(1)}=\frac{1}{2 k}+\mathcal{O}\left(\frac{1}{k^{2}}\right) \text {. }
$$

In fact,

$$
\frac{1}{2 k} \leq H_{k}^{(1)} \leq \frac{1}{2 k}+\mathcal{O}\left(\frac{1}{k^{2}}\right)
$$

In [9], it is shown that for the simple system, we have that $H_{k}^{(s)}$, the rate of $(s-1)$-stage HD-PIC, has an asymptotic scaling.

Theorem II.2: Fix $1 \leq s<\infty$ and assume that the powers are all equal. Then, as $k \rightarrow \infty$

$$
H_{k}^{(s)}=\frac{s}{8} \sqrt[s]{\frac{4}{k}}\left(1+\mathcal{O}\left(\frac{1}{\sqrt[s]{k}}\right)\right)
$$

In particular

$$
H_{k}^{(2)}=\frac{1}{2 \sqrt{k}}+\mathcal{O}\left(\frac{1}{k}\right)
$$

For $s=1,2$, the approximations $1 /(2 k)$ and $1 /(2 \sqrt{k})$ are quite accurate, as seen in Fig. 2. However, we see that for fixed $k$, the approximation becomes worse when $s$ increases.

It is clear that HD-PIC gives a significant increase in performance compared to the MF receiver, since $1 /(2 \sqrt{k})$ is much large than $1 /(2 k)$, so that $\exp (-n /(2 \sqrt{k}))$ is much smaller than 


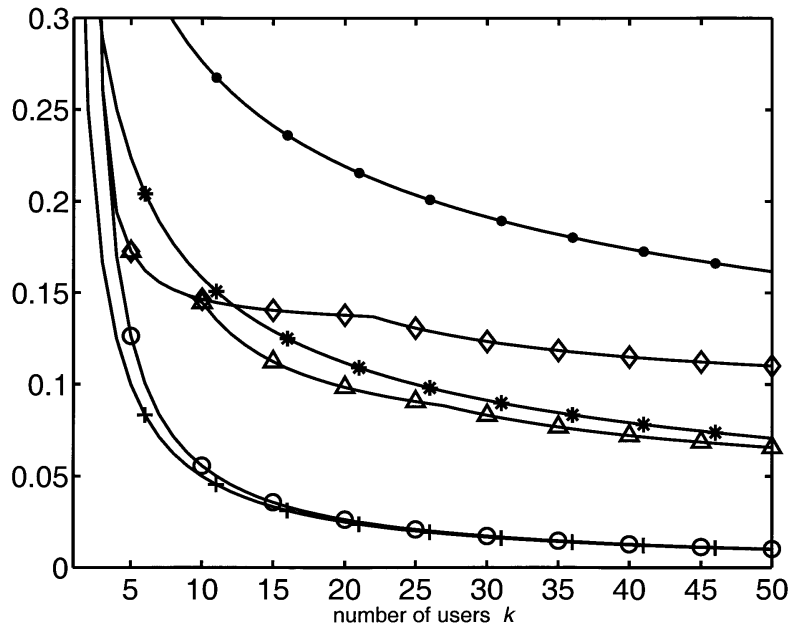

Fig. 2. Exponential rates $H_{k}^{(1)}, H_{k-}^{(2)}$, respectively, $H_{k}^{(3)}$ and the asymptotic behavior $\frac{1}{2 k}, \frac{1}{2 \sqrt{k}}$, respectively, $\frac{3}{8} \sqrt[3]{\frac{4}{k}}$. Here $\circ, \triangle, \diamond$ represent the exact values, and $+, *, \bullet$ the asymptotic values.

$\exp (-n /(2 k))$. Note the shift from $1 /(2 \cdot)$ to $1 /(2 \sqrt{\cdot})$. For example, in order to have approximately the same BEP, we can decrease the processing gain by a factor $\sqrt{k}$ or increase the number of users by a factor $\sqrt{k}$.

We will now give a heuristic explanation of Theorem II.2. We will start by explaining the result for $s=2$, and then comment on $s>2$. We will argue that

$$
H_{k, r}^{(2)} \approx \frac{r}{2 k}+\frac{1}{8 r} .
$$

Indeed, using (14), we then see that the minimum over $r$ is obtained when $r \approx \sqrt{k} / 2$, yielding $H_{k}^{(2)} \approx 1 /(2 \sqrt{k})$.

To see (19), note that $H_{k, r}^{(2)}$ is the rate of the event that

$$
Z_{2}^{(1)}<0, \ldots, Z_{r+1}^{(1)}<0, Z_{1}^{(2)}<0
$$

and all the other $Z_{j}^{(1)}>0$ for $j=r+2, \ldots, k$. When we know all the signs of the $Z^{(1)}$ 's, we can substitute these signs in the formula for $Z_{1}^{(2)}$. This yields that

$$
Z_{1}^{(2)}=1+\frac{2}{n} \sum_{j=2}^{r+1} \sum_{l=1}^{n} A_{1 l} A_{j l} .
$$

Note that $\mathbb{E}\left(Z_{j}^{(1)}\right)=1$ for all $j$. Therefore, the event $\left\{Z_{j}^{(1)} \geq 0\right\}$ is not a large deviation event. This explains why we can show that the events $Z_{j}^{(1)}>0$ for $j=r+2, \ldots, k$ do not contribute to the rate and we can leave these events out in the definition of $H_{k, r}^{(2)}$. Finally, independence of the sets $\left\{Z_{2}^{(1)}<0\right\}, \ldots,\left\{Z_{r+1}^{(1)}<0\right\}$, and $\left\{Z_{1}^{(2)}<0\right\}$ is clearly false for all finite $k$. However, it turns out to be asymptotically true for $k$ large, as shown in [9]. This yields that

$$
\begin{aligned}
H_{k, r}^{(2)} \approx \lim _{n \rightarrow \infty} \sum_{m=2}^{r+1}- & \frac{1}{n} \log \mathbb{P}\left(Z_{m}^{(1)}<0\right) \\
& \quad-\frac{1}{n} \log \mathbb{P}\left(1+\frac{2}{n} \sum_{j=2}^{r+1} \sum_{l=1}^{n} A_{1 l} A_{j l}<0\right)
\end{aligned}
$$

The first term produces $r H_{k}^{(1)} \approx r /(2 k)$, the second term can be computed to give $1 /(8 r)$ when $r$ is large. This gives an informal explanation of the asymptotic result in (19), and explains that to have one bit error at the first stage of HD-PIC, we need roughly $\sqrt{k} / 2$ bit errors in the matched filter stage. This explains why, in this setting, one stage of HD-PIC significantly decreases the BEP.

We finally give an informal explanation of Theorem II.2 for $s>2$. For a collection of indexes $R=\left(R_{\sigma}\right)_{\sigma=1}^{s}$, let $H_{k, R}^{(s)}$ denote the rate of the event that $\left\{Z_{m}^{(\sigma)}<0\right\}$ for all $m \in R_{\sigma}$, and $\left\{Z_{m}^{(\sigma)}>0\right\}$ for all $m \notin R_{\sigma}$. Thus, we have bit errors at stage $\sigma$ precisely for $m \in R_{\sigma}$. Then we have that

$$
H_{k}^{(s)}=\min _{R} H_{k, R}^{(s)}
$$

Let $r_{\sigma}^{(s)}=\left|R_{\sigma}^{(s)}\right|$. In [9], we show that we have

$$
H_{k, R}^{(s)} \approx \frac{r_{1}^{(s)}}{2 k}+\frac{r_{2}^{(s)}}{8 r_{1}^{(s)}}+\cdots+\frac{r_{s-2}^{(s)}}{8 r_{s-1}^{(s)}}+\frac{1}{8 r_{s-1}^{(s)}}
$$

Note that the right-hand side does not depend on the precise structure of $\left(R_{\sigma}^{(s)}\right)_{\sigma=1}^{s}$, but only on the sizes. The reason is that all dependence between $Z^{(\sigma)}$ at different stages is dictated by the signs of these quantities. After these signs are substituted, the random variables are asymptotically independent for large $k$, like in the case of $s=2$.

Minimization of the right hand side of (22) over $r_{1}^{(s)}, \ldots$, $r_{s-1}^{(s)}$ yields

$$
r_{\sigma}^{(s)} \approx\left(\frac{k}{4}\right)^{(s-\sigma) / s}
$$

Substitution yields (18).

We next describe the extension of the above results for the simple system with equal powers to the case where the powers are unequal, shown in [13]. For notational convenience, we introduce $P=\sum_{m=1}^{k} P_{m}$ and $P_{R}=\sum_{m \in R} P_{m}$, for sets $R \subset$ $\{1, \ldots, k\}$. For the MF system, the following result is proven for the exponential rate $H_{k}^{(1)}$.

Theorem II.3: For $P_{1} / P \rightarrow 0$

$$
H_{k ; 1}^{(1)}=\frac{P_{1}}{2 P}+\mathcal{O}\left(\left(\frac{P_{1}}{P}\right)^{2}\right) \text {. }
$$

Theorem II.3 is based on a Taylor expansion of the rate. For the HD-PIC system, it is more involved to find an asymptotic expansion, but it can still be done.

Theorem II.4: For $P_{1} / P \rightarrow 0$

$$
H_{k ; 1}^{(2)} \geq \frac{1}{2} \sqrt{\frac{P_{1}}{P}}+\mathcal{O}\left(\frac{P_{1}}{P}\right) .
$$

When there exists a $C>0$, such that, uniformly in $P / P_{1}$

$$
\min _{R}\left|\frac{P_{R}}{P_{1}}-\frac{1}{2} \sqrt{\frac{P}{P_{1}}}\right| \leq C\left(\frac{P}{P_{1}}\right)^{1 / 4}
$$


where the minimum ranges over all $R \subset\{2, \ldots, k\}$, equality is attained

$$
H_{k ; 1}^{(2)}=\frac{1}{2} \sqrt{\frac{P_{1}}{P}}+\mathcal{O}\left(\frac{P_{1}}{P}\right)
$$

A simple example where the above condition is satisfied is when $\max _{j, m} P_{j} / P_{m} \leq C$ for some $C$. We refer to [13] for more examples.

We have already seen that for the simple system $H_{k}^{(1)}$ and $H_{k}^{(2)}$ are given by

$$
H_{k}^{(1)} \approx \frac{1}{2 k} \quad \text { and } \quad H_{k}^{(2)} \approx \frac{1}{2 \sqrt{k}} .
$$

For the system with unequal powers, we see that, under some mild conditions on the powers

$$
H_{k ; 1}^{(1)} \approx \frac{1}{2} \frac{P_{1}}{P} \quad \text { and } \quad H_{k ; 1}^{(2)} \approx \frac{1}{2} \sqrt{\frac{P_{1}}{P}} .
$$

The same conclusion holds: interference cancellation significantly improves performance. The difference between equal and unequal powers is that $k$ is replaced by $P / P_{1}$. This also shows that the simple system is characteristic for the behavior of the more realistic system where unequal powers are incorporated; the natural substitution $k \rightarrow P / P_{1}$ suffices.

\section{Additive White Gaussian Noise (AWGN)}

Throughout the paper, we assume that no AWGN is present. At first sight, this will restrict the validity of the obtained results. However, we strongly believe that the results obtained in this paper are characteristic for the behavior of systems in which AWGN is present as long as there is very little noise, in the same way that the system with equal powers is characteristic for the system with unequal powers as described in the previous section. This belief stems from previous results obtained in [13]. We now explain how we can include AWGN in more detail, and define what we consider to be very little noise.

We include an AWGN term $n(t)$ in (3), i.e., we have that $\int_{0}^{t} n(s) d s$ is a Brownian motion. We assume that the two-sided spectrum of the AWGN equals $T_{c} \sigma^{2}$. Thus, the variance of the Brownian motion converges to 0 proportionally to $1 / n$ as $n \rightarrow \infty$. Using the above definitions, we see that $Z_{m}^{(1)}$ in (8) is changed to

$$
Z_{m}^{(1)}=P_{m}^{1 / 2}+\sum_{\substack{j=1 \\ j \neq m}}^{1 / 2} \frac{1}{n} \sum_{i=1}^{n} A_{j i} A_{m i}+\frac{1}{n} \sum_{i=1}^{n} A_{m i} N_{i}
$$

where $N_{i}$ are i.i.d. Gaussian random variables with mean 0 and variance $\sigma^{2}$. Alternatively, we can think of this power assumption as saying that the two-sided spectrum of the noise remains fixed, while all powers grow proportionally to $n$. This is necessary to make the AWGN of the same order as the multiple-access interference after averaging (see (8)), and makes sure that the large deviation methodology used in the present paper still applies. We note that PIC in general makes little sense when the AWGN is much larger than the multiple-access interference, and our bold assumption on the relation between the powers and the spectrum of the AWGN should be seen in this light. For a more detailed description of the model with AWGN, we refer to [13].

In [13], it is proven that under the same conditions of the powers as above, we have that

$$
H_{k ; 1}^{(1)} \approx \frac{1}{2} \frac{P_{1}}{P+\sigma^{2}} \quad \text { and } \quad H_{k ; 1}^{(2)} \approx \frac{1}{2} \sqrt{\frac{P_{1}}{P+\sigma^{2}}} .
$$

The above asymptotics is true whenever $\sigma^{2}$ is independent of the number of users $k$ and of the total power $P$. It is even allowed that $\sigma^{2}$ depends on $P$ and the corrections to the asymptotics in (25) are given. In other words, when we compare the system with unequal powers and AWGN with the system with equal powers and no AWGN, we need to replace $k$ by $\left(P+\sigma^{2}\right) / P_{1}$. The inclusion of AWGN increases the technicality of the proofs significantly, but eventually leads to the same conclusions. We expect that this extends to the case where $s>2$.

\section{E. Chernoff Bounds and Large $k$ Depending on $n$}

So far, we have only considered the asymptotic case $n \rightarrow$ $\infty$, while $k$ was fixed. In this subsection, we will prove results which hold for more general scenarios.

The Chernoff bound can be used to bound probabilities from above by an exponential, and is true regardless of $k, n$. For the MF system, the Chernoff bound is straightforward to calculate. The Chernoff bound is given by

$$
\mathbb{P}\left(\hat{b}_{10}^{(1)} \neq b_{10}\right) \leq e^{-n H_{k}^{(1)}}
$$

and this holds for any $n$ and $k$. Recall from (17) that $H_{k}^{(1)} \geq$ $1 /(2 k)$, so that $\mathbb{P}\left(\hat{b}_{10}^{(1)} \neq b_{10}\right) \leq e^{-n /(2 k)}$ for any $n$ and $k$. For the HD-PIC system, a similar statement can be derived. When we investigate, for example, the case of equal powers, we are able to prove

$$
\mathbb{P}\left(\hat{b}_{10}^{(2)} \neq b_{10}\right) \leq \sum_{r=1}^{k-1}\left(\begin{array}{c}
k-1 \\
r
\end{array}\right) e^{-n H_{k, r}^{(2)}} .
$$

The Chernoff bound is not tight in the sense that it approximates the BEP with any desired precision. Instead, it gives an upper bound in a very simple form. Note that if $n$ is large, the Chernoff bound is dominated by the term which has the smallest rate. Take, for example, $k=12$ and $n=63$. We can show that (see Table I), $H_{k, 2}^{(2)}$ minimizes $H_{k, r}^{(2)}$. Therefore, we expect that most of the sum is contributed by the second term. Substitution leads to

$$
\begin{aligned}
\mathbb{P}\left(\hat{b}_{10}^{(2)} \neq b_{10}\right) \leq & 1.32 \cdot 10^{-3}+1.76 \cdot 10^{-2}+5.28 \cdot 10^{-3} \\
& +3.44 \cdot 10^{-4}+\cdots \\
= & 2.45 \cdot 10^{-2} .
\end{aligned}
$$

The main contribution clearly comes from $r=2(72 \%)$, the contributions from $r=3$ and $r=1$ are also relevant (22\% and $5 \%)$, but the remainder of the sum is negligible (1\%).

We remark that the exponential rate and the Chernoff bound are closely related. Indeed, it is not possible to derive the Chernoff bound (valid for all $n$ and $k$ ) without knowing its large deviation properties (the asymptotic result for $n \rightarrow \infty$ ). This once more highlights the importance of the large deviation results. 


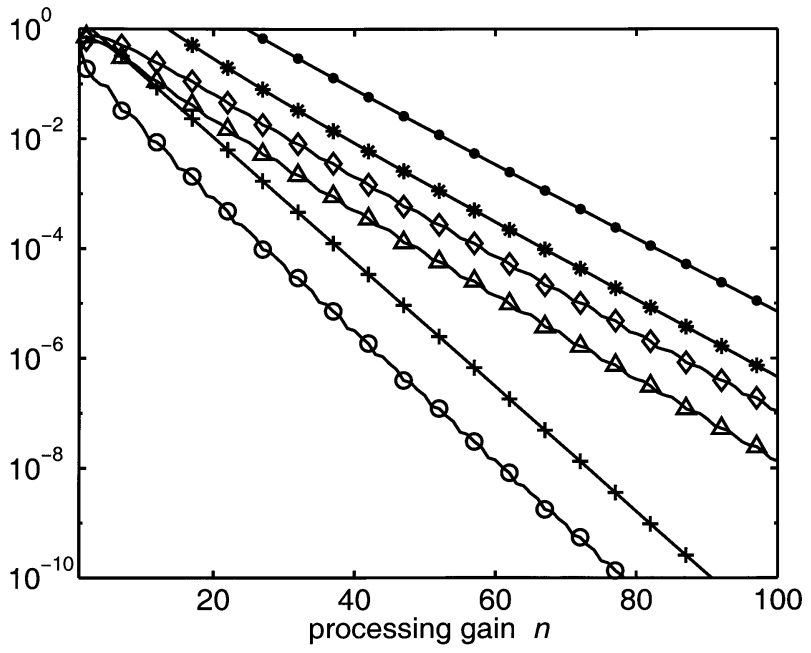

Fig. 3. BEP and Chernoff bound. The BEP for $k=3,6,9$ is marked with o, $\triangle$,, respectively. The Chernoff bound for $k=3,6,9$ is marked with $+, *, \bullet$, respectively.

In Fig. 3, simulated BEPs are compared with the Chernoff bound in (27) as a function of $n$. It is seen that the Chernoff bound indeed gives an upper bound. The exponential rate appears in this figure as the slope of the BEP and the Chernoff bound. The difference in slopes is very small, indicating that the large deviation approach is indeed promising for performance evaluation. These exact BEPs are obtained by an importance sampling procedure (see [14] and [15] for the details).

We next use the Chernoff bound in order to take $k$ large with $n$. From a practical point of view, one always wishes to have as many users as possible, so that the situation where $k$ is fixed and $n \rightarrow \infty$ may be violated. Instead, we now take $k=k_{n} \rightarrow \infty$. We prove the following result.

Theorem II.5: When all powers are equal and $k_{n} \rightarrow \infty$ such that $k_{n}=o\left(\frac{n}{\log n}\right)$

$$
\lim _{n \rightarrow \infty}-\frac{\sqrt[s]{k_{n}}}{n} \log \mathbb{P}\left(\hat{b}_{10}^{(s)} \neq b_{10}\right) \geq \frac{s}{8} \sqrt[s]{4} .
$$

The proof is given in Section V-B.

The above result implies in particular that when $k_{n} \rightarrow \infty$ such that $k_{n}=o\left(\frac{n}{\log n}\right)$ and we apply a single stage of HD-PIC, we have that $\mathbb{P}\left(\hat{b}_{10}^{(2)} \neq b_{10}\right)$ is to leading asymptotics equal to $\exp \left(-\frac{n}{2 \sqrt{k_{n}}}\right)$. This should be contrasted with the behavior that is expected when $k$ is of the order $n$. This is the central limit regime where we expect that the BEP converges as $n \rightarrow \infty$ to a nonzero constant. It pays to take $k$ slightly smaller than $n$ instead of a multiple of $n$, at least when considering a finite number of HD-PIC stages! In Section III-B, we will give a more precise (though nonrigorous) explanation of the central limit behavior and its relation to the HD-PIC results.

\section{F. Measures of Performance: SNR Versus Exponential Rate}

We next investigate the relation of the above result to the SNR, which is defined as

$$
\mathrm{SNR}=\frac{\mathbb{E}(Z)}{\sqrt{\operatorname{Var}(Z)}}
$$

where $Z$ denotes the decision statistic in the model under consideration. We consider the case $n \gg k \gg 1$ and $P_{m}=2$. We will show that in this case the SNR is not a good measure of performance, and therefore, we propose to use the exponential rate instead.

The asymptotic result for $H_{k}^{(1)}$ in (17) yields that the exponential rate of the BEP is approximately $1 /(2 k)$. Hence, the BEP is approximately

$$
\mathrm{BEP} \sim e^{-\frac{n}{2 k}}
$$

For the MF system, we know that SNR $=\sqrt{n /(k-1)}$, since

$$
\mathbb{E}\left(Z_{m}^{(1)}\right)=1 \quad \text { and } \operatorname{Var}\left(Z_{m}^{(1)}\right)=(k-1) / n .
$$

To approximate the BEP, one often substitutes the SNR in the well-known $Q$-function. Since (see [7])

$$
\frac{e^{-x^{2} / 2}}{\sqrt{2 \pi x^{2}}}\left(1-\frac{1}{x^{2}}\right)<Q(x)<\frac{e^{-x^{2} / 2}}{\sqrt{2 \pi x^{2}}},
$$

substituting the SNR yields the approximation

$$
\mathrm{BEP} \sim e^{-\mathrm{SNR}^{2} / 2}
$$

This approach gives the approximation

$$
\mathrm{BEP} \sim e^{-\frac{n}{2(k-1))}} \sim e^{-\frac{n}{2 k}}
$$

which agrees with the large deviation result in (29). Again, we see that our result extends to more general scenarios than the lightly loaded system, which was the starting point of the research.

For the simple system with one stage of HD-PIC, Theorem II. 2 that $H_{k}^{(2)} \approx 1 /(2 \sqrt{k})$, yielding

$$
\mathrm{BEP} \sim e^{-\frac{n}{2 \sqrt{k}}}
$$

in the case of a lightly loaded system.

However, for a system with one stage of HD-PIC, we show below that SNR $\geq \frac{e^{n / 4 k}}{(k-1)}$. Thus, substituting the the SNR into the $Q$-function results in

$$
\mathrm{BEP} \leq \exp \left\{-\frac{e^{\frac{n}{2 k}}}{2(k-1)^{2}}\right\}
$$

for all $n, k$. The latter value is far too small compared to the true asymptotics of the BEP in (30), which clearly indicates that the Gaussian approximation using the SNR is no good for lightly loaded system. We expect that our results extend to systems which are moderately loaded. The reasoning above can be adapted for other models, such as multistage HD-PIC or the optimal HD-PIC system described in what follows. Therefore, using the SNR together with a Gaussian approximation for such multiuser detection systems leads to faulty approximations for the BEP.

To prove the upper bound (31), observe that

$$
\mathbb{E}\left(\hat{b}_{1}^{(2)}\right)=1+2(k-1) \mathbb{P}\left(\hat{b}_{2}^{(1)} \neq b_{2}\right) \mathbb{E}\left(A_{21} A_{11} \mid \hat{b}_{2}^{(1)} \neq b_{2}\right) \approx 1,
$$

when $n$ is large compared to $k$ since $\mathbb{P}\left(\hat{b}_{2}^{(1)} \neq b_{2}\right) \approx 0$. Moreover

$$
\operatorname{Var}\left(Z_{1}^{(2)}\right) \leq \mathbb{E}\left(\left(Z_{1}^{(2)}-1\right)^{2}\right) \leq(k-1)^{2} \mathbb{P}\left(\hat{b}_{2}^{(1)} \neq b_{2}\right)
$$


where for the latter bound we use that $\left|A_{i j}\right|=1$. From the Chernoff bound (see (26)), and the fact that $H_{k}^{(1)} \geq \frac{1}{2 k}$ (see (17)), we end up with

$$
\operatorname{Var}\left(Z_{1}^{(2)}\right) \leq(k-1)^{2} e^{-\frac{n}{2 k}}
$$

This yields that

$$
\operatorname{SNR} \geq \frac{e^{\frac{n}{4 k}}}{(k-1)}
$$

so that

$$
e^{-\mathrm{SNR}^{2} / 2} \leq \exp \left\{-\frac{e^{\frac{n}{2 k}}}{2(k-1)^{2}}\right\}
$$

\section{ThE OPTIMAL HD-PIC SySTEM}

In this section, we show that after a finite number of stages of HD-PIC, the exponential rate of the BEP remains fixed. Then we study properties of this optimal hard-decision system, such as its exponential rate, and Chernoff bounds for the BEPs. Furthermore, we give results and intuition on the required number of stages to obtain or get close to this optimal system. The results show among other things that the BEP is exponentially small whenever $k \leq \delta n$ for some $\delta>0$ sufficiently small, which is quite remarkable.

\section{A. Optimal Hard Decisions}

We first list some easy consequences for the system with unequal powers. First, we define the worst case rate of a bit error to be

$$
H_{k}^{(s)}=\min _{m=1}^{k} H_{k ; m}^{(s)}
$$

Hence, $H_{k}^{(s)}$ is the exponential rate of the BEP of that user that has largest BEP.

Theorem III.1:

a) $H_{k}^{(s)}$ is monotone in $s$.

b) There exists an $s_{k} \leq 2^{k}+1$, such that $H_{k}^{(s)}=H_{k}^{\left(s_{k}\right)}$ for all $s \geq s_{k}$.

Theorem III.1 shows that we can speak of the system for $s=$ $s_{k}$ as the optimal system. For equal powers, we know that the rate for all users does not improve for $s \geq s_{k}$. For unequal powers, we can think of this system as having the optimal worse case rate, in the sense that the rate of the bit error minimized over all users is optimal.

We will use the definition of the sign function in (6). However, in the proof, we will comment on other definitions of the sign function where a similar result can be shown.

We will now prove the above theorem.

Proof of Theorem III.1: First, $s \mapsto H_{k}^{(s)}$ is nondecreasing. Indeed, to have a bit error, one of the interfering users has to have a bit error in the previous stage. Therefore, we have that for every $m$

$$
\mathbb{P}\left(\hat{b}_{m 0}^{(s)} \neq b_{m 0}\right) \leq \sum_{i=1}^{k} \mathbb{P}\left(\hat{b}_{i 0}^{(s-1)} \neq b_{i 0}\right) .
$$

Therefore, whenever $\log k=o(n)$

$$
H_{k ; m}^{(s)} \geq \min _{i=1, \ldots, k} H_{k ; i}^{(s-1)}=H_{k}^{(s-1)} .
$$

By taking the minimum over $m$ we indeed obtain that $H_{k}^{(s)} \geq$ $H_{k}^{(s-1)}$.

By the monotonicity above

$$
H_{k}^{(\infty)}=\lim _{s \rightarrow \infty} H_{k}^{(s)}
$$

exists (though it may be infinite). To see why $H_{k}^{(\infty)}$ with $k$ fixed is reached in a finite number of stages $s_{k}$ and $s_{k} \leq 2^{k}+1$, define

$$
R_{\sigma}^{(s)}=\left\{m: \operatorname{sgnr}_{m}\left(Z_{m}^{(\sigma)}\right) \neq 1\right\} .
$$

Then $R_{\sigma}^{(s)}$ is the set of bit errors at stage $s$ and stage $\sigma$, i.e., the set of indexes $j$ for which $\operatorname{sgnr}_{j}\left(Z_{j}^{(\sigma)}\right)<0$ when we enforce that $\operatorname{sgnr}_{m}\left(Z_{m}^{(s)}\right)<0$. We observe that necessarily $R_{\sigma^{\prime}}^{(s)}=R_{\sigma^{\prime \prime}}^{(s)}$, for some $\sigma^{\prime}<\sigma^{\prime \prime} \leq 2^{k}+1$. Indeed, there are at most $2^{k}$ configurations $R_{\sigma}$, so that after at most $2^{k}+1$ stages, one of the configurations is assumed twice. Then $Z_{m}^{\left(\sigma^{\prime \prime}+1\right)} \equiv Z_{m}^{\left(\sigma^{\prime}+1\right)}$ for all $1 \leq m \leq k$ and thus $R_{\sigma^{\prime}+1}^{(s)}=R_{\sigma^{\prime \prime}+1}^{(s)}$. Therefore, $R_{\sigma+i\left(\sigma^{\prime \prime}-\sigma\right)}^{(s)}=R_{\sigma}^{(s)}$ for all $\sigma^{\prime} \leq \sigma<\sigma^{\prime \prime}$ and all $i \in \mathbb{N} \cup\{0\}$. By (7), all stages beyond $\sigma^{\prime \prime}$ are determined by the stages $1, \ldots, \sigma^{\prime \prime}$. Clearly, for every stage $s^{\prime}$ with $s \geq s^{\prime}>\sigma^{\prime \prime}$, some users have bit errors. Thus, the described scenario that forces a bit error in stage $s$ also forces a bit error in stage $s^{\prime}>s$. Since $H_{k}^{\left(s^{\prime}\right)}$ is the worst case rate over all scenarios and all users, it follows that $H_{k}^{\left(s^{\prime}\right)} \leq H_{k}^{(s)}$. By the fact that $H_{k}^{(s)}$ is nondecreasing, we necessarily have that $H_{k}^{\left(s^{\prime}\right)}=H_{k}^{(s)}$ for all $s^{\prime} \geq \sigma^{\prime \prime}$. Since $\sigma^{\prime \prime} \leq s \leq 2^{k}+1$, the desired statement follows.

The statement of Theorem III.1 can be extended to other definitions of the sign function. For example, when we let the sign of 0 to be 0 , then we can copy the original proof, apart from the fact that we let $R_{\sigma ; 0}^{(s)}$ be the set of values $m$ where $Z_{m}^{(\sigma)}=0$, and $R_{\sigma ; 1}^{(s)}$ the set where $Z_{m}^{(\sigma)}<0$. Then, when $s \geq 3^{k}+1$, we must have $R_{\sigma^{\prime} ; i}^{(s)}=R_{\sigma^{\prime \prime} ; i}^{(s)}$, for $i=0,1$ and some $\sigma^{\prime}<\sigma^{\prime \prime} \leq 3^{k}+1$. This again implies that $H_{k}^{(s)}=H_{k}^{\left(s_{k}\right)}$ for some $s_{k}$.

For the system with equal powers, we are also allowed to use a sign function that assumes values \pm 1 independently every time it is used, rather than fixing it per user. Indeed, the limit determining $H_{k}^{(\infty)}$ exists, so we can reach the limit just by using the odd $n$. In this case, we cannot have $Z_{m}^{(\sigma)}=0$, except when $\sigma=1$. Therefore, every user draws at most one time a random sign function, which makes the statement equivalent to the statement with our original definition of sgnr. For unequal powers, this argument unfortunately does not hold. However, $\mathbb{Q}$ is dense in $\mathbb{R}$, so that for almost all powers $\left(P_{1}, \ldots, P_{k}\right)$ and for all integers $n, Z_{m}^{(\sigma)} \neq 0$. This explains why the problems arising from the definition of the sign of zero are somewhat academic.

Before turning to the investigation of this optimal HD-PIC system, we relate the results to the well-known results for the Hopfield model. HD-PIC is an example of a Hopfield neural network in which the bits are updated in a parallel fashion. For such Hopfield models with integer weights, it is well known that there is a limit cycle of length at most two. Moreover, 
bounds are given for the number of stages needed to arrive in this cycle. See, e.g., [4] and the references therein. For HD-PIC, the weights depend on the square roots of the powers. Therefore, the assumption of integer weights puts restriction on the allowed powers. Moreover, in the Hopfield literature, the sign of zero is always assumed to be zero. Therefore, we have chosen to give a self-contained proof using the trivial fact that a map mapping $\{-1,1\}^{k}$ into $\{-1,1\}^{k}$ makes a cycle in at most $2^{k}+1$ iterations, and the cycle then has length at most $2^{k}$. It would be of great interest to investigate in more detail what is the implication for the performance of HD-PIC of the fact that the limit cycle has length at most 2 . Possibly some of our results can be improved.

We will next investigate this optimal HD-PIC system. Recall that because of the hard decisions, the decisions at stage $\sigma$ only depend on the decisions at stage $\sigma-1$. For illustration purposes, we will first investigate the system with two users. We will use that when user 1 has no bit error at stage $\sigma$, user 2 necessarily has no bit error at stage $\sigma+1$. We denote a bit error by $\square$ and no bit error by $\bullet$ in Fig. 4 . All possible five scenarios (except the trivial scenario where no errors at all are made, which does not contribute to the BEP) are shown.

It is clear that within three stages, the set where bit errors are made becomes periodic. We will call this a periodic scenario. A periodic scenario has a big "advantage" over nonperiodic scenarios. As long as the scenario is not yet periodic, specifying which users have bit errors at a certain stage results in an increase of the exponential rate of the BEP. Indeed, in the case where $n$ is large and the number of users fixed, users do not tend to have a bit error since it is more likely to estimate a bit correctly. However, in a periodic scenario, this is not true any more. For example, to get a bit error for user 1 at stage $s=1001$, it is sufficient to specify the positions of bit errors in stages 1,2 , and 3 (the first and last scenario in Fig. 4 will do). From that point onwards, the bit errors are determined by the bit errors in the first three stages.

Two essentially different scenarios are characterizing the behavior of the optimal system for $k=2$. The first is the so-called disjoint scenario, where at every stage user 1 has a bit error and user 2 not, or vice versa. The other scenario, which we will call the overlapping scenario is the scenario where at every stage both user 1 and user 2 have a bit error. Note that for both the disjoint as well as the overlapping scenario, the periodic behavior kicks in at stage 1 . For both scenarios, we can calculate the exponential rate. The minimum of the two exponential rates indicates which scenario typically is observed, and this rate is the exponential rate of the BEP for $k=2$. The above provides an explanation of the observed periodicity when $k=2$. We next turn to an investigation of the optimal HD-PIC system when $k \geq 3$ and extend the above observations to this case.

When $k \geq 3$, we extend the above periodic scenarios in the following way. For every $r$, at stage $1,3,5, \ldots$, bit errors are made for users in some set $R_{1}$, with $\left|R_{1}\right|=r$. At stage $2,4,6, \ldots$, bit errors are made for users in the set $R_{2}$ with $\left|R_{2}\right|=r$. When $R_{1} \cap R_{2}=\varnothing$, we speak of the disjoint scenario. Whenever $R_{1}=R_{2}$, we will call it the overlapping scenario. All other scenarios are called partly overlapping.

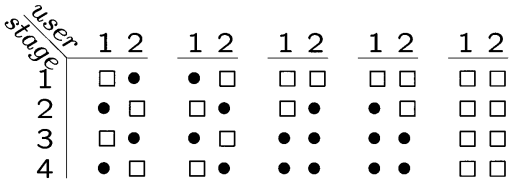

Fig. 4. Possible scenarios for the HD-PIC model with two users.

TABLE II

EXPECTED SCENARIO FOR OPTIMAL HD-PIC MODEL

\begin{tabular}{clcc}
\hline$k$ & $r$ & $k$ & $r$ \\
\hline $1-30$ & $1(\mathrm{~d})^{1}$ & $337-414$ & $10(\mathrm{o})$ \\
$31-73$ & $2(\mathrm{~d})$ & $415-499$ & $11(\mathrm{o})$ \\
$74-83$ & $3(\mathrm{~d})$ & $500-592$ & $12(\mathrm{o})$ \\
$84-107$ & $5(\mathrm{o})^{2}$ & $593-694$ & $13(\mathrm{o})$ \\
$108-153$ & $6(\mathrm{o})$ & $695-803$ & $14(\mathrm{o})$ \\
$154-206$ & $7(\mathrm{o})$ & $804-920$ & $15(\mathrm{o})$ \\
$207-267$ & $8(\mathrm{o})$ & $920-1000$ & $16(\mathrm{o})$ \\
$268-336$ & $9(\mathrm{o})$ & & \\
\hline
\end{tabular}

${ }^{1}$ (d) means disjoint scenario has the smallest exponential rate

${ }^{2}$ (o) means overlapping scenario has the smallest exponential rate.

The statement of Theorem III.1b) can be understood by noting that after at most $2^{k}+1$ stages, the set where bit errors are made becomes periodic. We expect that the scenarios that we typically observe are periodic already at stage 1 , similarly to the case where $k=2$, as we will now argue heuristically. Indeed, specifying the bit errors at the initial nonperiodic stages makes the exponential rate of the BEP larger, while for the periodic stages we do not need to specify the positions of bit errors, as these are determined by the first few stages. The longer the number of stages where the behavior is not yet periodic, the larger the exponential rate of the BEP and thus this behavior is less typical. This suggests that the scenarios that we typically observe are either the disjoint, the partly overlapping, or the overlapping scenario. The overlapping scenario has period 1 , while the other scenarios have period 2, so stretching the above heuristic even further, it is tempting to assume that the overlapping scenario gives the smallest exponential rate, and is therefore typical. However, this is not true when $k$ is small (see also item 2) in the following).

We have numerically computed the rates of the three different scenarios for $k=1, \ldots, 1000$, and we observe the following phenomena (see Table II).

1) The partly overlapping scenario never has the smallest exponential rate, so that this scenario is less likely than the overlapping or disjoint scenario. It seems that both extremes (the overlapping and disjoint scenarios) do a better job, since a smaller exponential rate means, for $n$ large, a larger BEP.

2) For small $k$, the disjoint scenario has the smallest exponential rate. We now give a heuristic explanation for this fact. For $r$ fixed, in the disjoint scenario, a user at stage 2 has contribution from $r$ noise terms, whereas for the overlapping scenario the user has contribution from only $r-1$ terms (the user does not interfere with his own signal). For larger $k$, however, the overlapping scenario has the smallest exponential rate, which is due to the fact that the interference terms are more strongly positively correlated 


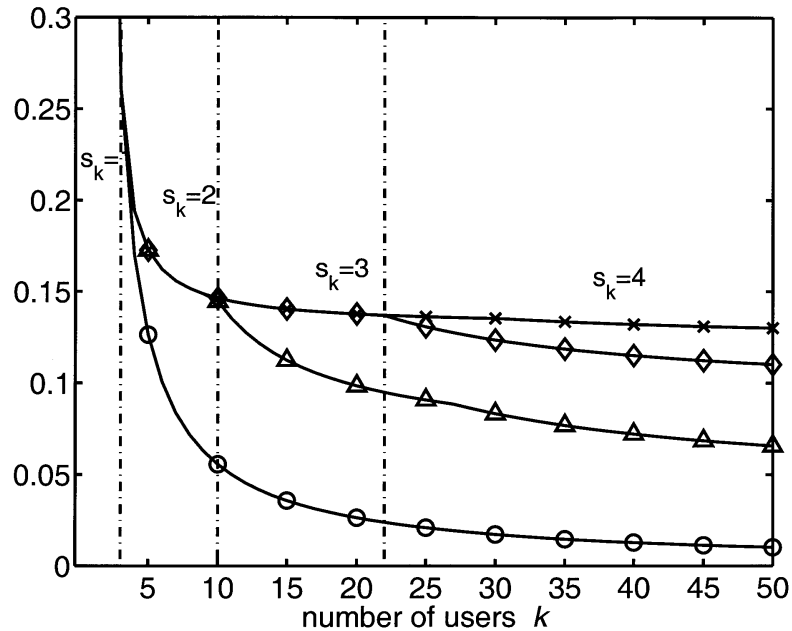

Fig. 5. The exponential rates $H_{k}^{\left(s_{k}\right)}$ and $H_{k}^{(s)}$ for $s=1,2,3$.

in the overlapping scenario than in the disjoint scenario. For the case $k \rightarrow \infty$, it is implicitly proven in the proof of Theorem III.2 a) that the overlapping scenario indeed has the smallest exponential rate, and the partly overlapping scenario has a strictly larger exponential rate.

3) For $k \rightarrow \infty$, also $r \rightarrow \infty$, but much slower than $k$. The numerical results indicate that $r \approx \sqrt{k} / 2$.

To illustrate 2) and 3), the optimal $r$ is shown for $k=1-1000$ in Table II. Also, it is indicated whether the disjoint or the overlapping scenario has the smallest exponential rate.

In Fig. 5, the numerical values for the exponential rates $H_{k}^{\left(s_{k}\right)}$ are given, together $H_{k}^{(s)}$ for $s=1,2,3$. The results for $s=3$ are obtained using similar techniques as in Theorem II.1. However, we have not stated the result for $s=3$ in this paper. The rate $H_{k}^{\left(s_{k}\right)}$ is, in fact, the rate corresponding to the disjoint scenario for $r=1$ or $r=2$. For $k=3$, it is seen that $H_{3}^{(1)}=H_{3}^{(2)}=H_{3}^{(3)}$, so that $s_{3}=1$. For $4 \leq k \leq 9$, we see $H_{k}^{(1)}<H_{k}^{(2)}=H_{k}^{(3)}$, so that $s_{k}=2$. We see that one stage of HD-PIC gives an improvement in exponential rate. However, adding one more stage does not result in any improvement. For $10 \leq k \leq 22$, two-stage HD-PIC gives an improvement over one-stage HD-PIC. The numerical result show that there is a block scenario (in fact, the disjoint scenario with $r=1$ ) with the same rate. Therefore, $s_{k}=3$ in this case. For $23 \leq k \leq 50$, we expect $s_{k}=4$, even though we cannot calculate $H_{k}^{(4)}$ analytically.

We now turn to a lower bound of the exponential rate for the optimal system. Clearly, (18) has no meaning for $s \rightarrow \infty$, but assuming (18) and the monotonicity of $s \mapsto H_{k}^{(s)}$ it follows for the simple system that for all $\epsilon>0, k^{\epsilon} H_{k}^{(\infty)} \rightarrow \infty$ when $k \rightarrow \infty$. Thus, if $H_{k}^{(\infty)}$ converges to 0 as $k \rightarrow \infty$, it does so slower than any power of $1 / k$. The following theorem states that the exponential rate of the optimal system remains strictly positive as $k \rightarrow \infty$, and we identify the limiting rate under certain mild assumptions on the powers $P_{1}, \ldots, P_{m}$ that we will define now. We will assume that $\left(\mathcal{P}_{1}-\mathcal{P}_{4}\right)$ hold, where the conditions $\left(\mathcal{P}_{1}-\mathcal{P}_{4}\right)$ are defined by

$\left(\mathcal{P}_{1}\right)$ there exists a $\delta>0$ s. t. $\sharp\left\{j: P_{j} \in[\delta, 1 / \delta]\right\} \rightarrow \infty$;

$\left(\mathcal{P}_{2}\right) \lim _{k \rightarrow \infty} k P^{-1}<\infty$;

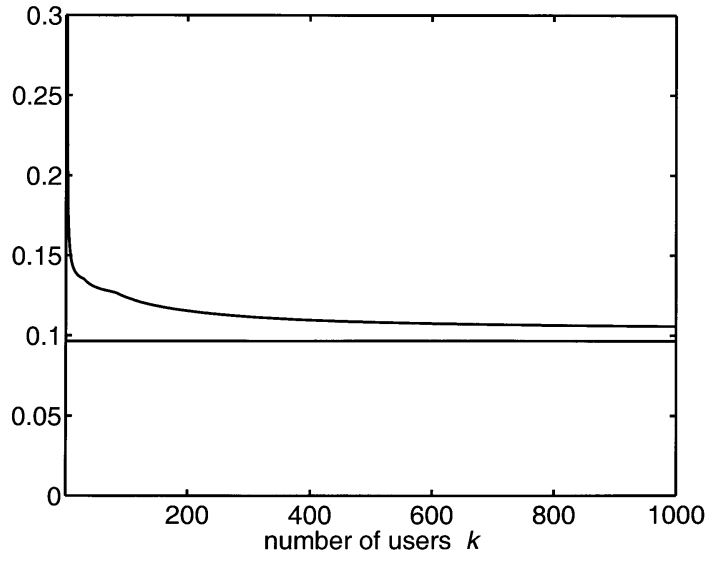

Fig. 6. Upper and lower bounds for the exponential rates $H_{k}^{\left(s_{k}\right)}$.

$$
\begin{aligned}
& \left(\mathcal{P}_{3}\right) k^{-1} P^{\max } \rightarrow 0 \\
& \left(\mathcal{P}_{4}\right) k P^{\min } \rightarrow \infty
\end{aligned}
$$

Here $P^{\max }=\max _{m} P_{m}, P^{\min }=\min _{m} P_{m}$. The main result in this section is the following theorem.

\section{Theorem III.2:}

a) For the general system, for all $s>2^{k}$

$$
H_{k}^{(s)} \geq \frac{1}{2} \log 2-\frac{1}{4} \approx 0.09657 .
$$

For the system with equal powers the bound is obtained for $s>k$.

b) If the powers are such that $\left(\mathcal{P}_{1}-\mathcal{P}_{4}\right)$ hold, then

$$
\lim _{k \rightarrow \infty} H_{k}^{\left(s_{k}\right)}=\frac{1}{2} \log 2-\frac{1}{4} .
$$

We stress that the case of equal powers is covered in Theorem III.2 b).

In Fig. 6, an upper and a lower bound are given for the exponential rate $H_{k}^{\left(s_{k}\right)}$ with equal powers. The lower bound follows from Theorem III.2 a), whereas the upper bound is obtained by evaluating the minimal exponential rates of the disjoint, overlapping, and partly overlapping scenarios in Table II. It is possible that some other scenario yields the minimal exponential rate, so that this indeed is only an upper bound. However, we expect that the upper bound is, in fact, equal to $H_{k}^{\left(s_{k}\right)}$, but we lack a proof of this fact.

The proof of the preceding result will be given in the subsequent sections. We will start with bounds on moment-generating functions in Section IV. These bounds will be used in the proof of Theorem III.2 in Section V.

\section{B. Chernoff Bounds and Large $k$ Depending on $n$}

In Section V-B, we prove the following Chernoff bound for the optimal system.

Theorem III.3: For $s>2^{k_{n}}$

$$
\mathbb{P}\left(\hat{b}_{10}^{(s)} \neq b_{10}\right) \leq 8^{k_{n}} e^{-I n}
$$

where $I=\frac{1}{2} \log 2-\frac{1}{4}$. 
When the powers are equal, the same result holds when $s>$ $k_{n}$ and in the upper bound the factor $8^{k_{n}}$ can be replaced by $\left(k_{n}+1\right) 4^{k_{n}}$.

We emphasize that the factor $8^{k_{n}}$ is a combinatorial factor that arises in the bounds in the proof. We believe that the bound could be improved by a more careful analysis.

The above result shows that the limit of $k$ and $n$ can be taken simultaneously, instead of the order common in large deviation theory (first $n \rightarrow \infty$, and subsequently $k \rightarrow \infty$ ). Moreover, using the theorem above, we find the following corollary.

Corollary III.4: If $k_{n} \leq \delta n$ with $\delta<I /(3 \log 2) \approx 0.04644$ and $s>2^{k_{n}}$, then $\mathbb{P}\left(\hat{b}_{10}^{(s)} \neq b_{10}\right)$ is exponentially small for $n \rightarrow \infty$.

When the powers are equal, the same result holds when $s>k_{n}$, and $\delta<I /(2 \log 2) \approx 0.06966$.

Corollary III.4 follows immediately from Theorem III.3 by noting that the upper bound is monotone in $k_{n}$, and substitution of $k_{n}=\delta n$ yields

$$
\mathbb{P}\left(\hat{b}_{10}^{(s)} \neq b_{10}\right) \leq e^{-(I-3 \delta \log 2) n}
$$

which is indeed exponentially small whenever $\delta<I /(3 \log 2)$. The improvement for equal powers follows similarly. We note here that by taking the number of possible bit errors in the first stage into account, that in the case of equal powers we can extend the result to $\delta \leq 0.11$. We will not give that proof here.

Since, for $k=\mathcal{O}(n)$, we have central limit behavior, the proposition above implies that applying HD-PIC sufficiently often enables us to reduce the BEP from $\mathcal{O}(1)$ for the MF system, to exponentially small for the optimal HD-PIC system. We now give a more extensive heuristic explanation for this effect.

We take $k=\delta n$ for some constant $\delta>0$. We assume that the powers are equal, and for simplicity take $P_{m}=1$. In this case, we have that by the central limit theorem, $Z_{m}^{(1)} \approx \mathcal{N}(1, \delta)$. Therefore, the BEP is approximately equal to the probability that an $\mathcal{N}(1, \delta)$ is less than 0 . Hence, the BEP is approximately $\epsilon^{(1)}=Q(1 / \sqrt{\delta})$. Therefore, we expect $\epsilon^{(1)} \delta n$ bit errors in the first stage $(s=1)$. We stress here that we are allowed to use the central limit theorem since $s$ is fixed and $k=\delta n$, so that the BEP converges as $n \rightarrow \infty$. Therefore, the problems that we demonstrate with the use of the central limit theorem in Section II-F do not arise.

Now, given that there are $\epsilon^{(1)} \delta n$ bit errors in the first stage, it is natural to assume that $Z_{m}^{(2)} \approx \mathcal{N}\left(1,4 \epsilon^{(1)} \delta\right)$. The above is only a heuristic argument though, as the fact that we have $\epsilon^{(1)} \delta n$ bit errors introduces dependence between $\left\{Z_{m}^{(1)}\right\}_{m=1}^{k}$ and $\left\{Z_{m}^{(2)}\right\}_{m=1}^{k}$ and the corresponding different coding sequences. Continuing the above reasoning, we expect that the BEP is approximately

$$
\epsilon^{(2)}=Q\left(1 /\left(2 \sqrt{\epsilon^{(1)} \delta}\right)\right)
$$

and we expect $\epsilon^{(2)} \delta n$ bit errors in the second stage $(s=2)$. Repeating the above heuristic argument, we find that, with

$$
\epsilon^{(s)}=Q\left(1 /\left(2 \sqrt{\epsilon^{(s-1)} \delta}\right)\right)
$$

we expect approximately $\epsilon^{(s)} \delta n$ bit errors in the $s$ th stage.
The above heuristic predicts that the BEP converges when $s$ remains fixed and $n \rightarrow \infty$ with $k=\delta n$, which is what one would expect and is observed in practice. However, the naive Gaussian assumption used in the above argument is flawed, as shown for example in [3], where improved Gaussian assumptions are presented. The flaw resides in the strong dependence between different interference terms.

From Theorem II.2 combined with Theorem III.2, we do know that $s_{k} \rightarrow \infty$ when $k \rightarrow \infty$. Therefore, the argument showing convergence of the BEP to a nonzero limit does not apply for the optimal HD-PIC system, and we even see in Theorem III.3 that the optimal HD-PIC system has a much smaller BEP than the BEP after any finite number of stages whenever $\delta>0$ is sufficiently small.

\section{On the Number of Stages to Optimality}

In this subsection, we will investigate the number of stages necessary to reach the asymptotic optimal rate $\frac{1}{2} \log 2-\frac{1}{4}$. We will prove the following theorem. In its statement, we will recall that $P=\sum_{m} P_{m}$ and $P^{\min }=\min _{m} P_{m}$.

Theorem III.5: For the general system, for all $0<\epsilon<$ $\frac{1}{2} \log 2-\frac{1}{4}$ and for all $s$ such that $s>\left\lceil\epsilon^{-1} \log \frac{P}{P^{\min }}\right\rceil$

$$
H_{k}^{(s)} \geq \frac{1}{2} \log 2-\frac{1}{4}-\epsilon .
$$

Consequently, for all $s>\left\lceil\epsilon^{-1} \log \left(\frac{P}{P^{\min }}\right)\right\rceil$ and all $k_{n}$

$$
\mathbb{P}\left(\hat{b}_{10}^{(s)} \neq b_{10}\right) \leq\left(\left\lceil\epsilon^{-1} \log \left(\frac{P}{P^{\min }}\right)\right]+1\right) 4^{k_{n}} e^{-(I-\epsilon) n} .
$$

We know that $I=\frac{1}{2} \log 2-\frac{1}{4}$ is the asymptotically optimal rate for the system where all the powers are equal. Note that when all powers are comparable (e.g., when $\max _{j, m} \frac{P_{j}}{P_{m}}<C$ for some constant $C<\infty$ uniformly in $k$ ), then $\epsilon^{-1} \log \frac{P}{P^{\min }} \sim$ $\epsilon^{-1} \log k$. Theorem III.5 states that when we apply at least that many stages of HD-PIC, then the limiting rate will be asymptotically bounded from below by $I$. This suggests that $s_{k}$ grows roughly like $\log k$. It is an interesting problem to determine more precisely how $s_{k}$ grows. It follows from Theorem III.2 together with Theorem II.2 that $s_{k} \rightarrow \infty$. However, it would be of practical importance to know the precise rate, or even an upper bound on $s_{k}$. We have the following conjecture.

Conjecture III.6: For the general system

$$
\limsup _{k \rightarrow \infty} \frac{\log s_{k}}{\log \log k}=1 \text {. }
$$

Conjecture III.6 says that $\frac{s_{k}}{\log k}$ cannot grow or decrease faster than any small power of $\log k$.

Theorem III.5, together with Theorem III.2, suggest that $s_{k} \leq \epsilon_{k}^{-1} \log k$ for every $\epsilon_{k} \downarrow 0$. We believe that, in fact, a logarithmic number of stages is required to obtain the optimal HD-PIC system. However, we have no proof for this belief. This belief stems from the fact that we expect the proof of Theorem II.2 to hold for some $s$ that tend to infinity with $k$ sufficiently slowly. More precisely, we expect that the strategy described in Section II-C remains true for as long as $s_{k} \leq(\log k)^{1-\epsilon}$ for any $\epsilon>0$. The reason is that in (23), there is an essential 
change when $s \ll \log k$ compared to $s_{k}=\mathcal{O}(\log k)$, in the sense that for the former

$$
\frac{r_{\sigma}^{(s)}}{r_{\sigma+1}^{(s)}} \rightarrow \infty
$$

whereas for the latter, the above converges to a constant. When this convergence is toward a constant, we cannot expect (22) to be a good approximation. Moreover, note that when $s=$ $(\log k)^{1-\epsilon}$, then substitution of $s$ into the right-hand side of (18) gives

$$
\frac{s}{8} e^{-\log k / s}=\frac{(\log k)^{1-\epsilon}}{8} e^{-(\log k)^{\epsilon}} \rightarrow 0
$$

for all positive $\epsilon$. This is clearly far away from the rate of the optimal HD-PIC system in Theorem III.2. Therefore, we believe $s_{k} \geq(\log k)^{1-\epsilon}$ for all $\epsilon>0$, which explains Conjecture III.6.

We have the following corollary to Theorem III.5.

Corollary III.7: Fix $\epsilon>0$ such that $\epsilon<2 \delta \log 2-I$. If

a) $k_{n} \leq \delta n$ with $\delta<I /(2 \log 2) \approx 0.06966$;

b) $s>\left\lceil\epsilon^{-1} \log \left(\frac{P}{P^{\min }}\right)\right\rceil$;

c) $\log \log \left(\frac{P}{P^{\min }}\right)=o(n)$;

then $\mathbb{P}\left(\hat{b}_{10}^{(s)} \neq b_{10}\right)$ is exponentially small for $n \rightarrow \infty$.

The proof of Corollary III.7 follows from (42) together with a similar argument as in (40). We note that the assumption in c) is extremely weak. We need it to make sure that $\left\lceil\epsilon^{-1} \log \left(\frac{P}{P^{\min }}\right)\right\rceil$ is not exponentially large in $n$. Corollary III.7 shows that under mild condition on the powers, the BEP is exponentially small whenever $s$ is sufficiently large when $k_{n}=\delta n$ and $\delta<0.06966$. Thus, it improves Corollary III.4 substantially in that the number of stages can be taken much smaller $\left(\left\lceil\epsilon^{-1} \log \left(\frac{P}{P^{\min }}\right)\right\rceil\right.$ which tends to be of order $\log k_{n}$ under mild conditions on the powers, compared to $2^{k_{n}}+1$ ), and that larger $k_{n}$ are allowed.

Corollary III.7 implies that in a moderately loaded system and when all powers are comparable, order $\log k$ stages of HD-PIC is sufficient to decrease the BEP from $O(1)$ to exponentially small.

\section{Bounds On Moment Generating Functions}

In this section, we will give sharp bounds on certain moment-generating functions that will prove to be essential in the analysis of the optimal HD-PIC system.

We define

$$
S_{n}=\sum_{i=1}^{n} P_{i}^{1 / 2} X_{i}
$$

where

$$
\mathbb{P}\left(X_{i}= \pm 1\right)=\frac{1}{2} .
$$

We will also use the notation $P=\sum_{i=1}^{n} P_{i}$. The main result of this section is as follows.
Proposition IV.1:

a) For all $n \in \mathbb{N}, u \in \mathbb{R}$, and all $0 \leq v \leq \frac{1}{P}$

$$
\mathbb{E}\left[e^{u S_{n}+\frac{v}{2} S_{n}^{2}}\right] \leq \frac{e^{\frac{P u^{2}}{2(1-P v)}}}{\sqrt{1-P v}} .
$$

b) For all $-\frac{1}{P} \leq v \leq \frac{1}{P}$

$$
\mathbb{E}\left[e^{\frac{v}{2} S_{n}^{2}}\right] \leq \frac{1}{\sqrt{1-P v}} .
$$

Note that if $n$ is large, then $S_{n} \approx P^{1 / 2} Z$, where $Z$ has a standard normal distribution. The bounds in (45) and (46) show that the moment-generating function of $S_{n}$ and $S_{n}^{2}$, for the appropriate ranges of the variables $u$ and $v$, are at least bounded from above by the moment-generating functions of $P^{1 / 2} Z$ and $P Z^{2}$, $e^{P u^{2} / 2}$ and $1 / \sqrt{1-P v}$, respectively.

Proof of Proposition IV.1 a): This bound is easiest. Let $Z$ have a standard normal distribution, then we know that $\mathbb{E}\left(e^{v Z}\right)=e^{v^{2} / 2}$. Hence, we get that

$$
\begin{aligned}
\mathbb{E}\left[e^{u S_{n}+\frac{v}{2} S_{n}^{2}}\right] & =\mathbb{E}\left[e^{(u+\sqrt{v} Z) S_{n}}\right] \\
& =\mathbb{E}\left[\prod_{i=1}^{n} \cosh \left(P_{i}^{1 / 2}(u+\sqrt{v} Z)\right)\right] .
\end{aligned}
$$

We use $\cosh t \leq e^{t^{2} / 2}$ to arrive at

$$
\begin{aligned}
\mathbb{E}\left[e^{u S_{n}+\frac{v}{2} S_{n}^{2}}\right] & \leq \mathbb{E}\left[\prod_{i=1}^{n} e^{\frac{P_{i}}{2}(u+\sqrt{v} Z)^{2}}\right] \\
& =e^{\frac{P u^{2}}{2}} \mathbb{E}\left[e^{P u \sqrt{v} Z+\frac{v P Z^{2}}{2}}\right] .
\end{aligned}
$$

We complete the proof by noting that, for $v \leq \frac{1}{P}$

$$
\mathbb{E}\left[e^{P u \sqrt{v} Z+\frac{v P Z^{2}}{2}}\right]=\frac{e^{\frac{P^{2} u^{2} v}{2(1-P v)}}}{\sqrt{1-P v}}
$$

and by rearranging terms.

Proof of Proposition IV.1 b): The claim for $0 \leq v \leq \frac{1}{P}$ follows from Proposition IV.1 a) proved already. The claim for $v<0$ is more difficult, and we will use induction on $n$. Define

$$
f_{n}(v)=\mathbb{E}\left[e^{\frac{v}{2} S_{n}^{2}}\right] .
$$

The induction hypothesis is that

$$
f_{n}(v) \leq \frac{1}{\sqrt{1-P v}}, \quad \text { for all }-\frac{1}{P} \leq v \leq 0 .
$$

Clearly, for $n=0$, the above is trivial, as both the left- and right-hand sides are equal to 1 . We next advance the induction. We write

$$
\begin{aligned}
f_{n}(v) & =\mathbb{E}\left[e^{\frac{v}{2} S_{n}^{2}}\right]=e^{\frac{v P_{n}}{2}} \mathbb{E}\left[e^{\frac{v}{2} S_{n-1}^{2}+P_{n}^{1 / 2} v S_{n-1} X_{n}}\right] \\
& =e^{\frac{v P_{n}}{2}} \mathbb{E}\left[e^{\frac{v}{2} S_{n-1}^{2}} \cosh \left(P_{n}^{1 / 2} v S_{n-1}\right)\right] .
\end{aligned}
$$

We again use $\cosh t \leq e^{t^{2} / 2}$ to arrive at

$$
f_{n}(v) \leq e^{\frac{v P_{n}}{2}} \mathbb{E}\left[e^{\frac{v+P_{n} v^{2}}{2} S_{n-1}^{2}}\right]=e^{\frac{v P_{n}}{2}} f_{n-1}\left(v+P_{n} v^{2}\right) .
$$

To prove the claim, we first show that for $-\frac{1}{P} \leq v \leq 0$ we have

$$
-\frac{1}{P-P_{n}} \leq v+P_{n} v^{2} \leq 0
$$

Indeed, since $-\frac{1}{P} \leq v \leq 0$, we have $0 \leq 1+P_{n} v \leq 1$, so that $-1 \leq\left(-1-P_{n} v\right)\left(1+P_{n} v\right) \leq\left(P v-P_{n} v\right)\left(1+P_{n} v\right)$

$$
=\left(P-P_{n}\right) v\left(1+P_{n} v\right) \leq 0
$$


where the last inequality follows from $P-P_{n} \geq 0$ and $v \leq 0$. Thus, (49) follows. We, therefore, can substitute the induction hypothesis (48) for $n-1$, so that it remains to show that

$$
\frac{e^{v P_{n} / 2}}{\sqrt{1-\left(P-P_{n}\right)\left(v+P_{n} v^{2}\right)}} \leq \frac{1}{\sqrt{1-P v}} .
$$

Since $e^{t} \geq 1+t+t^{2} / 2$ for all $t \geq 0$

$$
e^{v P_{n} / 2}=\frac{1}{\sqrt{e^{-v P_{n}}}} \leq \frac{1}{\sqrt{1-P_{n} v+\frac{P_{n}^{2} v^{2}}{2}}} .
$$

Multiplying and rearranging terms gives

$$
\begin{aligned}
(1- & \left.P_{n} v+\frac{P_{n}^{2} v^{2}}{2}\right)\left(1-\left(P-P_{n}\right)\left(v+P_{n} v^{2}\right)\right) \\
& =1-P v+\frac{P_{n}^{2} v^{2}}{2}\left(1+P v-P_{n} v\left(1+\left(P-P_{n}\right) v\right)\right) \\
& \geq 1-P v
\end{aligned}
$$

since $1+P v \geq 0, v \leq 0$, and $1+\left(P-P_{n}\right) v \geq 0$. This completes the proof.

\section{PROOFS}

In this section we will first prove Theorems III.2 a) and III.5, where we will use Proposition IV.1. Then, we will prove the Chernoff bounds in Theorems II.5 and III.3 in Section V-B. This proof makes use of the proof of Theorem III.2 a). Finally, we prove Theorem III.2 b) for equal powers. The proof of Theorem III.2 b) for unequal powers satisfying the power conditions $\left(\mathcal{P}_{1}-\mathcal{P}_{4}\right)$ will be given in the Appendix.

\section{A. Proof of Theorem III.2 a) and Theorem III.5}

In this subsection, we will prove Theorems III.2 a) and III.5 simultaneously. For completeness, we will repeat the statements. For $s=2^{k}+1$

$$
H_{k}^{(s)} \geq \frac{1}{2} \log 2-\frac{1}{4} \text {. }
$$

For every

$$
0<\epsilon<I=\frac{1}{2} \log 2-\frac{1}{4} .
$$

For $s=\left\lceil\epsilon^{-1} \log \left(P / P^{\text {min }}\right)\right\rceil+1$

$$
H_{k}^{(s)} \geq \frac{1}{2} \log 2-\frac{1}{4}-\epsilon .
$$

By the fact that $H_{k}^{(s)}$ is decreasing in $k,(50)$ and (51) are equivalent to Theorems III.2 a) and III.5, respectively. In Section III, we have shown that there is an optimal HD-PIC system, and that for $s \geq 2^{k}+1$, the set of bit errors is periodic. For any set $A \subset\{1, \ldots, k\}$, we let $P_{A}=\sum_{m \in A} P_{m}$. When $s=2^{k}+1$, there must be a $\sigma \leq 2^{k}+1$ such that $P_{R_{\sigma}^{(s)}} \geq P_{R_{\sigma-1}^{(s)}}$. In fact, when the powers are equal, we have that $P_{R_{\sigma}^{(s)}}=P\left|R_{\sigma}^{(s)}\right|$, and the above must happen already when $s \geq k+1$. We focus on that stage $\sigma$ and are only interested in the event $\left\{P_{R_{\sigma}^{(s)}} \geq P_{R_{\sigma-1}^{(s)}}\right\}$.

Furthermore, when $s=\left\lceil\epsilon^{-1} \log \left(P / P^{\min }\right)\right\rceil+1$, there must be a $\sigma \leq\left\lceil\epsilon^{-1} \log \left(P / P^{\min }\right)\right\rceil+1$ such that

$$
P_{R_{\sigma}^{(s)}} \geq(1-\epsilon) P_{R_{\sigma-1}^{(s)}} .
$$

Indeed, when this is not the case after

$$
\sigma-1=\left\lceil\epsilon^{-1} \log \left(P / P^{\min }\right)\right\rceil
$$

stages, we have

$$
P_{R_{\sigma-1}^{(s)}} \leq(1-\epsilon)^{\sigma-1} P_{R_{0}^{(s)}} \leq \exp \left(\frac{\log (1-\epsilon)}{\epsilon} \log \left(\frac{P}{P^{\min }}\right)\right) P .
$$

Since $1-\epsilon \leq e^{-\epsilon}$, also $\log (1-\epsilon) / \epsilon \leq-1$, so that

$$
P_{R_{\sigma-1}^{(s)}} \leq \exp \left(-\log \left(\frac{P}{P^{\min }}\right)\right) P=P^{\min } .
$$

However, always $P_{R_{\sigma}^{(s)}} \geq P^{\mathrm{min}}$, so that

$$
P_{R_{\sigma}^{(s)}} \geq(1-\epsilon) P_{R_{\sigma-1}^{(s)}}
$$

so certainly after at most $s=\left\lceil\epsilon^{-1} \log \left(P / P^{\text {min }}\right)\right\rceil+1$ stages the desired event has occurred. We focus on the stage $\sigma$ at which the desired event occurs and are only interested in that occurrence.

At this point, we remark that once we obtain for, $P_{R_{\sigma}^{(s)}} \geq$ $(1-\epsilon) P_{R_{\sigma-1}^{(s)}}$, that

$$
-\lim _{n \rightarrow \infty} \frac{1}{n} \log \mathbb{P}\left(P_{R_{\sigma}^{(s)}} \geq(1-\epsilon) P_{R_{\sigma-1}^{(s)}}\right) \geq \frac{1}{2} \log 2-\frac{1}{4}-\epsilon
$$

we immediately obtain the result for $\epsilon=0$, which is the statement (50).

We use that when $A=R_{\sigma-1}^{(s)}$ and $B=R_{\sigma}^{(s)}$, that then for all $m \in B$, we have that

$$
\frac{1}{2}\left(2 P_{m}\right)^{1 / 2} Z_{m}^{(\sigma)}=P_{m}+\frac{2}{n} \sum_{l=1}^{n} \sum_{j \in A \backslash\{m\}}\left(P_{j} P_{m}\right)^{1 / 2} A_{j l} A_{m l} .
$$

As the number of configurations $\left(R_{\sigma}^{(s)}\right)_{\sigma=1}^{s}$ is finite, and we have $P_{R_{s}^{(s)}}>0$ for all $\sigma \leq s$, we obtain that $H_{k}^{(s)}$ is bounded from below by the minimum over $A$ and $B$ such that $P_{B} \geq$ $(1-\epsilon) P_{A}>0$ of

$$
\begin{aligned}
& -\lim _{n \rightarrow \infty} \frac{1}{n} \\
& \cdot \log \mathbb{P}\left(\sum_{j \in A} \sum_{l=1}^{n}\left(P_{j} P_{m}\right)^{1 / 2} A_{j l} A_{m l}+\frac{n P_{m}}{2} \leq 0 \forall m \in B\right) \\
& \geq-\lim _{n \rightarrow \infty} \frac{1}{n} \log \mathbb{P}\left(\sum_{\substack{j \in A \\
m \in B \backslash\{j\}}} \sum_{l=1}^{n}\left(P_{j} P_{m}\right)^{1 / 2} A_{j l} A_{m l}+\frac{n P_{B}}{2} \leq 0\right)
\end{aligned}
$$

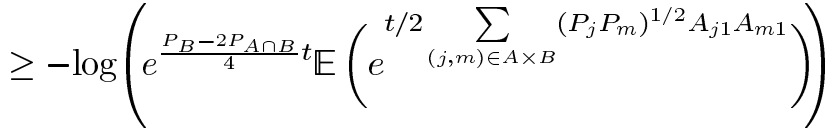

where the last inequality is the exponential Chebycheff's inequality for $t \leq 0$. We write $S_{A}=\sum_{j \in A} P_{j}^{1 / 2} A_{j 1}$ to end up with

$$
H_{k}^{(s)} \geq \min _{\substack{A, B \\ P_{B} \geq(1-\epsilon) P_{A}>0}}-\log \left(\mathbb{E}\left(e^{(t / 2) S_{A} S_{B}}\right)\right)+\frac{P_{B}-2 P_{A \cap B}}{4} t .
$$

We will now bound the moment-generating function from below using Proposition IV.1. Since we still have freedom to choose $t$, we restrict $t$ to satisfy

$$
-\min \left\{\frac{3}{2 P_{B}}, \frac{1}{P_{A \cap B}}\right\} \leq t \leq 0 .
$$

Note that when (53) holds (we only need $t \geq-3 /\left(2 P_{B}\right)$ here), we have

$$
1+\left(P_{B \backslash A}+P_{A \backslash B}\right) \frac{t}{4} \geq 1+\frac{P_{B} t}{4}\left(1+\frac{1}{1-\epsilon}\right) \geq 0
$$


and

$$
\frac{P_{A \backslash B} P_{B \backslash A}}{4} t^{2} \leq \frac{P_{B}^{2}}{4(1-\epsilon)} t^{2} \leq 1
$$

for $\epsilon \leq 7 / 16$, which is implied by $\epsilon \leq I$.

We first write $S_{A}=S_{A \cap B}+S_{A \backslash B}$, and we use the fact that $S_{A \backslash B}$ is independent from $\left(S_{B}, S_{A \cap B}\right)$ to get that $\mathbb{E}\left(e^{\frac{t}{2} S_{B} S_{A}}\right)$ equals

$$
\begin{aligned}
\mathbb{E}\left(e^{\frac{t}{2} S_{B} S_{A \cap B}} e^{\frac{t}{2} S_{B} S_{A \backslash B}}\right) & \\
= & \mathbb{E}\left(e^{\frac{t}{2} S_{B} S_{A \cap B}} \prod_{j \in A \backslash B} \cosh \left(\frac{t}{2} P_{j}^{1 / 2} S_{B}\right)\right) \\
\leq & \mathbb{E}\left(e^{\frac{t}{2} S_{B} S_{A \cap B}} e^{\frac{t^{2}}{8} P_{A \backslash B} S_{B}^{2}}\right) .
\end{aligned}
$$

We write the right hand side of (56) as

$$
\mathbb{E}\left(e^{\left(\frac{t}{2}+\frac{t^{2}}{8} P_{A \backslash B}\right) S_{A \cap B}^{2}} e^{\left(\frac{t}{2}+\frac{t^{2}}{4} P_{A \backslash B}\right) S_{A \cap B} S_{B \backslash A}+\frac{t^{2}}{8} P_{A \backslash B} S_{B \backslash A}^{2}}\right)
$$

and use Proposition IV.1 a) with $u=\left(\frac{t}{2}+\frac{t^{2}}{4} P_{A \backslash B}\right) S_{A \cap B}$ and $0 \leq v=\frac{t^{2}}{4} P_{A \backslash B} \leq 1 / P_{B \backslash A}$ (use (55)) to bound the expectation over $S_{B \backslash A}$ as

$$
\begin{aligned}
& \frac{1}{\sqrt{1-P_{A \backslash B} P_{B \backslash A} t^{2} / 4}} \\
& \times \mathbb{E}\left(e^{\left(\frac{t}{2}+\frac{t^{2}}{8} P_{A \backslash B}\right) S_{A \cap B}^{2}} e^{P_{B \backslash A} \frac{\left(t / 2+P_{A \backslash B} t^{2} / 4\right)^{2}}{2\left(1-P_{A} \backslash B_{B} P_{B} \backslash A^{\left.t^{2} / 4\right)}\right.} S_{A \cap B}^{2}}\right) \\
& =\frac{1}{\sqrt{1-P_{A \backslash B} P_{B \backslash A} t^{2} / 4}} \mathbb{E}\left(e^{\tilde{t} S_{A \cap B}^{2} / 2}\right) \\
& \leq \frac{1}{\sqrt{1-P_{A \backslash B} P_{B \backslash A} t^{2} / 4}} \frac{1}{\sqrt{1-P_{A \cap B} \tilde{t}}} .
\end{aligned}
$$

Here, the last inequality is valid as long as $|\tilde{t}| \leq \frac{1}{P_{B \cap A}}$, where

$$
\begin{aligned}
\tilde{t} & =t+\frac{t^{2} P_{A \backslash B}}{4}+P_{B \backslash A} \frac{\left(\frac{t}{2}+P_{A \backslash B} t^{2} / 4\right)^{2}}{1-P_{A \backslash B} P_{B \backslash A} t^{2} / 4} \\
& =\frac{t+\left(P_{B \backslash A}+P_{A \backslash B}\right) t^{2} / 4}{1-P_{A \backslash B} P_{B \backslash A} t^{2} / 4} .
\end{aligned}
$$

From the first line in (57), it is clear that $\tilde{t} \geq t$, so that restriction (53) directly implies $\tilde{t} \geq-1 /\left(P_{A \cap B}\right)$. From the second expression in (57), together with $t \leq 0$ and (54) it follows that $\tilde{t} \leq 0$. We proceed by multiplying the two square roots to obtain

$$
\mathbb{E}\left(e^{\frac{t}{2} S_{A} S_{B}}\right) \leq \frac{1}{\sqrt{1-t P_{B \cap A}-\frac{t^{2}}{4}\left(P_{A} P_{B}-P_{A \cap B}^{2}\right)}}
$$

since

$$
P_{A \backslash B} P_{B \backslash A}+P_{A \cap B}\left(P_{A \backslash B}+P_{B \backslash A}\right)=P_{A} P_{B}-P_{A \cap B}^{2} .
$$

Substituting (58) into (52) yields that whenever (53) holds, we have that $H_{k}^{(s)}$ is bounded from below by

$$
\begin{aligned}
& \min _{A, B} \quad \frac{1}{2} \log \left(1-t P_{A \cap B}-\frac{t^{2}}{4}\left(P_{A} P_{B}-P_{A \cap B}^{2}\right)\right) \\
& P_{B} \geq(1-\epsilon) P_{A}>0 \\
& -\frac{P_{B}-2 P_{A \cap B}}{4} t .
\end{aligned}
$$

Since $t=-\frac{1}{P_{B}}$ satisfies the restrictions in (53), we can substitute this value to obtain

$$
\begin{array}{r}
H_{k}^{(s)} \geq \min _{\substack{A, B \\
P_{B} \geq(1-\epsilon) P_{A}>0}} \frac{1}{2} \log \left(1-\frac{P_{A}}{4 P_{B}}+\frac{P_{A \cap B}}{P_{B}}+\frac{P_{A \cap B}^{2}}{4 P_{B}^{2}}\right) \\
\\
\quad-\frac{1}{4}\left(2 \frac{P_{A \cap B}}{P_{B}}-1\right) .
\end{array}
$$

It is clear that this lower bound of $H_{k}^{(s)}$ is decreasing in $P_{A}$ and that $P_{A} \leq \frac{1}{1-\epsilon} P_{B}$. Therefore, substituting $P_{A}=\frac{1}{1-\epsilon} P_{B}$ still gives a lower bound. Clearly, $\frac{P_{A \cap B}}{P_{B}} \in[0,1]$. The above lower bound is, therefore, attained at

$$
\begin{aligned}
\min _{0 \leq \alpha \leq 1} \frac{1}{2} \log \left(1-\frac{1}{4(1-\epsilon)}+\alpha+\frac{\alpha^{2}}{4}\right) & -\frac{1}{4}(2 \alpha-1) \\
& =\min _{0 \leq \alpha \leq 1} f(\alpha) .
\end{aligned}
$$

Differentiating $f(\alpha)$ with respect to $\alpha$ gives that $f^{\prime}(\alpha)$ equals

$$
-\frac{1}{2}+\frac{1}{2} \frac{1+\frac{\alpha}{2}}{1-\frac{1}{4(1-\epsilon)}+\alpha+\frac{\alpha^{2}}{4}}=\frac{\frac{1}{4(1-\epsilon)}-\frac{\alpha}{2}-\frac{\alpha^{2}}{4}}{1-\frac{1}{4(1-\epsilon)}+\alpha+\frac{\alpha^{2}}{4}} .
$$

Hence,

$$
f^{\prime}(\alpha)>0, \quad \text { for } \alpha<\sqrt{1+1 /(1-\epsilon)}-1
$$

and

$$
f^{\prime}(\alpha)<0, \quad \text { ffor } \alpha>\sqrt{1+1 /(1-\epsilon)}-1 .
$$

Therefore, the minimum of $f$ is attained at either $\alpha=0$ or at $\alpha=1$. Substitution yields that

$$
\begin{aligned}
f(0) & =\frac{1}{2} \log \left(1-\frac{1}{4(1-\epsilon)}\right)+\frac{1}{4} \\
& =\frac{1}{2} \log \frac{3}{4}+\frac{1}{4}+\frac{1}{2} \log \left(1-\frac{1}{3}\left(\frac{1}{1-\epsilon}-1\right)\right)
\end{aligned}
$$

and

$$
\begin{aligned}
f(1) & =\frac{1}{2} \log \left(\frac{9}{4}-\frac{1}{4(1-\epsilon)}\right)-\frac{1}{4} \\
& =\frac{1}{2} \log 2-\frac{1}{4}+\frac{1}{2} \log \left(1-\frac{1}{8}\left(\frac{1}{1-\epsilon}-1\right)\right) .
\end{aligned}
$$

Finally, observe that for $0 \leq \epsilon \leq 1 / 2$, we have $1 /(1-\epsilon) \leq$ $1+2 \epsilon$, and for $0 \leq \epsilon \leq 2 / 3$ we have

$$
e^{-2 \epsilon} \leq 1-2 \epsilon+2 \epsilon^{2} \leq 1-2 \epsilon+\frac{4 \epsilon}{3}=1-\frac{2 \epsilon}{3}
$$

so that $\log (1-2 \epsilon / 3) \leq-2 \epsilon$. Substituting this yields

$$
\begin{array}{r}
\log \left(1-\frac{1}{3}\left(1-\frac{1}{1-\epsilon}\right)\right) \geq-2 \epsilon \\
\log \left(1-\frac{1}{8}\left(1-\frac{1}{1-\epsilon}\right)\right) \geq-\frac{3}{4} \epsilon \geq-2 \epsilon .
\end{array}
$$

Therefore, since $\frac{1}{2} \log \frac{3}{4}+1 / 4>\frac{1}{2} \log 2-1 / 4$

$$
H_{k}^{(s)} \geq \min \{f(0), f(1)\}=\frac{1}{2} \log 2-\frac{1}{4}-\epsilon .
$$

This completes the proof of Theorem III.5. Substituting $\epsilon=0$ gives Theorem III.2 a). 


\section{B. Proof of the Chernoff Bounds}

We will start by proving Theorem II.5 for $s=2$. The extension to $s>2$ will follow later, and is a small adaptation of the proof for $s=2$.

We have that

$$
\begin{aligned}
& \mathbb{P}\left(\operatorname{sgnr}_{1}\left(Z_{1}^{(2)}\right)<0\right) \leq \mathbb{P}\left(Z_{1}^{(2)} \leq 0\right) \\
& \quad \leq \sum_{r=1}^{k_{n}-1}\left(\begin{array}{c}
k_{n}-1 \\
r
\end{array}\right) \mathbb{P}\left(Z_{1}^{(2)} \leq 0, \underset{m=2}{r+1} Z_{m}^{(1)} \leq 0, \underset{m=r+2}{k_{n}} Z_{m}^{(1)} \geq 0\right) .
\end{aligned}
$$

We split the sum over $r$ into two parts: $1 \leq r \leq 4 \sqrt{k_{n}}$ and $r>4 \sqrt{k_{n}}$. We start with the first term. The standard Chernoff bound gives

$$
\mathbb{P}\left(Z_{1}^{(2)} \leq 0, \underset{m=2}{r+1} Z_{m}^{(1)} \leq 0, \min _{m=r+2}^{k_{n}} Z_{m}^{(1)} \geq 0\right) \leq e^{-n H_{k_{n}, r}^{(2)}} .
$$

We bound, using $\left(\begin{array}{c}k_{n}-1 \\ r\end{array}\right) \leq k_{n}^{r}$ and $e^{-n H_{k_{n}, r}^{(2)}} \leq e^{-n H_{k_{n}}^{(2)}}$

$$
\begin{aligned}
\sum_{r=1}^{4 \sqrt{k_{n}}}\left(\begin{array}{c}
k_{n}-1 \\
r
\end{array}\right) e^{-n H_{k_{n}, r}^{(2)}} & \leq k_{n}^{4 \sqrt{k_{n}}+1} e^{-n H_{k_{n}}^{(2)}} \\
& =e^{4 \frac{k_{n} \log k_{n}}{\sqrt{k_{n}}}+\frac{\sqrt{k_{n}} \log k_{n}}{\sqrt{k_{n}}}} e^{-n H_{k_{n}}^{(2)}} .
\end{aligned}
$$

The first term on the right-hand side is $e^{o\left(\frac{n}{\sqrt{k_{n}}}\right)}$, since $k_{n}=o\left(\frac{n}{\log n}\right)$ implies $k_{n} \log k_{n}=o(n)$. Therefore, this term is bounded from above by

$$
e^{o\left(\frac{n}{\sqrt{k_{n}}}\right)} e^{-n H_{k_{n}}^{(2)}}=e^{o\left(\frac{n}{\sqrt{k_{n}}}\right)} e^{-\frac{n}{2 \sqrt{k_{n}}}(1+o(1))} .
$$

This proves that the first term has the right order, and it remains to show that the other term is an error term. In order to do this, we will first prove the following lemma.

Lemma V.1: For every $k, n$

$$
\mathbb{P}\left(Z_{1}^{(2)} \leq 0, \underset{m=2}{r+1} Z_{m}^{(1)} \leq 0, \min _{m=r+2}^{k} Z_{m}^{(1)} \geq 0\right) \leq e^{-\frac{r}{4 k} n} .
$$

Proof: We bound, using $\mathbb{P}(A \cap B) \leq \mathbb{P}(A)$

$$
\begin{aligned}
\mathbb{P}\left(Z_{1}^{(2)} \leq 0, \underset{m=2}{r+1}{ }_{m} Z_{m}^{(1)}\right. & \left.\leq 0, \min _{m=r+2}^{k} Z_{m}^{(1)} \geq 0\right) \\
& \leq \mathbb{P}\left(\max _{m=1}^{r} Z_{m}^{(1)} \leq 0\right) \leq \mathbb{P}\left(\sum_{m=1}^{r} Z_{m}^{(1)} \leq 0\right) .
\end{aligned}
$$

We can compute that

$$
\sum_{m=1}^{r} Z_{m}^{(1)}=\frac{1}{n} \sum_{l=1}^{n} \sum_{m=1}^{r} A_{m l} \sum_{i=1}^{k} A_{i l},
$$

Therefore, by the exponential Chebycheff inequality, for every $t \leq 0$, the probability of interest is bounded by

$$
\left(\min _{t \leq 0} \mathbb{E}\left(e^{\frac{t}{2} S_{r} S_{k}}\right)\right)^{n} \leq\left(\min _{t \leq 0} \mathbb{E}\left(e^{\frac{t}{2} S_{r}^{2}} e^{\frac{t}{2} S_{r}\left(S_{k}-S_{r}\right)}\right)\right)^{n}
$$

where we write $S_{m}=\sum_{l=1}^{m} A_{1 l}$. We next bound the momentgenerating function from above. We first use the independence of $S_{r}$ and $S_{k}-S_{r}$ to obtain

$$
\mathbb{E}\left(e^{\frac{t}{2} S_{r}^{2}} e^{\frac{t}{2} S_{r}\left(S_{k}-S_{r}\right)}\right)=\mathbb{E}\left(e^{\frac{t}{2} S_{r}^{2}} \cosh \left(\frac{t}{2} S_{r}\right)^{k-r}\right) .
$$

We next use the bound $1 \leq \cosh (t) \leq e^{t^{2} / 2}$ to bound (63) as

$$
\mathbb{E}\left(e^{\frac{t}{2} S_{r}^{2}} \cosh \left(\frac{t}{2} S_{r}\right)^{k}\right) \leq \mathbb{E}\left(e^{\left(t+\frac{k t^{2}}{4}\right) S_{r}^{2} / 2}\right) .
$$

As long as $t+\frac{k t^{2}}{4} \geq-\frac{1}{r}$, we can use Proposition IV.1 b) to obtain

$$
\mathbb{E} e^{\frac{t}{2} S_{r}^{2}} e^{\frac{t}{2} S_{r}\left(S_{k}-S_{r}\right)} \leq \frac{1}{\sqrt{1-r t-\frac{r k t^{2}}{4}}}
$$

Therefore, we arrive at

$$
\begin{aligned}
\mathbb{P}\left(Z_{1}^{(2)} \leq 0, \max _{m=2}^{r+1} Z_{m}^{(1)}\right. & \left.\leq 0, \min _{m=r+2}^{k} Z_{m}^{(1)} \geq 0\right)^{1 / n} \\
\leq & \min _{t \leq 0} \exp \left(-\frac{1}{2} \log \left(1-r t-\frac{r k t^{2}}{4}\right)\right) .
\end{aligned}
$$

The optimal $t$ is attained at $t=-2 / k$. For this choice, we have that $t+k t^{2} / 4=-1 / k \geq-1 / r$. This justifies (65). We further observe that for $t=-2 / k$, we have

$$
\log \left(1-r t-\frac{r k t^{2}}{4}\right)=\log \left(1+\frac{r}{k}\right) .
$$

Finally, observe that $\frac{1}{2} \log (1+x) \geq x / 4$ for all $0 \leq x \leq 1$, which completes the proof of the lemma.

We now complete the proof of Theorem II.5 for $s=2$ using Lemma V.1. Since $k_{n} e^{-\frac{n}{4 k_{n}}}=o(1)$, the sum over $r$ satisfying $r>4 \sqrt{k_{n}}$ is bounded by

$$
\begin{aligned}
\sum_{r>4 \sqrt{k_{n}}} k_{n}^{r} e^{-\frac{n r}{4 k_{n}}} & =\left(e^{\log k_{n}-\frac{n}{4 k_{n}}}\right)^{4 \sqrt{k_{n}}} \sum_{r>0}\left(e^{\log k_{n}-\frac{n}{4 k_{n}}}\right)^{r} \\
& \leq 2 \exp \left(4 \sqrt{k_{n}} \log k_{n}-\frac{n}{\sqrt{k_{n}}}\right) .
\end{aligned}
$$

This satisfies the required bound since $\sqrt{k_{n}} \log k_{n}=o\left(\frac{n}{\sqrt{k_{n}}}\right)$, so that we have

$$
\begin{aligned}
\mathbb{P}\left(\operatorname{sgnr}_{1}\left(Z_{1}^{(2)}\right)<0\right) & \leq e^{-\frac{n}{2 \sqrt{k_{n}}}(1+o(1))}+2 e^{-\frac{n}{\sqrt{k_{n}}}(1+o(1))} \\
& =e^{-\frac{n}{2 \sqrt{k_{n}}}(1+o(1))} .
\end{aligned}
$$

The proof for $s>2$ is similar, and we point out the differences only. Write $a_{s}=\frac{s}{8} \sqrt[s]{4}$. We can copy the proof in (66) and (67) to show that the probability that there are at least $c_{1} k_{n}^{(s-1) / s}$ bit errors in the first stage is bounded above by $k_{n}^{c_{1} k_{n}^{(s-1) / s}} e^{-2 n k_{n}^{-1 / s} a_{s}}$ whenever $c_{1}>0$ is large enough, which is of lower order. Therefore, we only have to deal with the case where there are at most $c_{1} k_{n}^{(s-1) / s}$ bit errors in the first stage, and we restrict ourselves to this case.

In this case, an easy extension of the proof of Lemma V.1 shows that the probability that there are at least $c_{2} k_{n}^{(s-2) / s}$ bit-errors in the second stage is bounded above by $k_{n}^{c_{2} k_{n}^{(s-2) / s}} e^{-2 n k_{n}^{-1 / s} a_{s}}$ whenever $c_{2}>0$ is large enough. Because we can show that this is again of lower order, we may assume that there are at most $c_{2} k_{n}^{(s-2) / s}$ bit errors in the second stage. We can repeat this argument for the stages $3, \ldots, s-1$, so that we only have to deal with the probability that user 0 has a bit error in stage $s$, intersected by the events 
$\left|R_{\sigma}^{(s)}\right| \leq c_{\sigma} k_{n}^{(s-\sigma) / s}$. We now can use the standard Chernoff bound to end up with

$$
\begin{aligned}
\mathbb{P}\left(Z_{1}^{(s)} \leq 0\right) \leq & s e^{-2 n k_{n}^{-1 / s} a_{s}} \\
& +\mathbb{P}\left(\forall \sigma=1, \ldots, s: 1 \leq\left|R_{\sigma}^{(s)}\right| \leq c_{\sigma} k_{n}^{(s-\sigma) / s}\right) \\
\leq & e^{-n H_{k_{n}}^{(s)}} \prod_{i=1}^{s}\left(\begin{array}{c}
k_{n} \\
c_{\sigma} k_{n}^{(s-\sigma) / s}
\end{array}\right)+s e^{-2 n k_{n}^{-1 / s} a_{s}} \\
\leq & e^{-n k_{n}^{-1 / s} a_{s}(1+o(1))}+s e^{-2 n k_{n}^{-1 / s} a_{s}} .
\end{aligned}
$$

This completes the proof for $s>2$.

We next turn to the proof of Theorem III.3 and the Chernoff bound in (42), which is quite easy when we use results of the proof of Theorem III.2 in Section V-A. In the proof, we have used the fact that for $s \geq 2^{k_{n}}+1$, there must be a stage $\sigma$ such that $P_{R_{\sigma}^{(s)}} \geq P_{R_{\sigma-1}^{(s)}}$. The rate of this event is proven to be bounded from below by $I=\frac{1}{2} \log 2-\frac{1}{4}$. The number of possible stages at which this can happen is $2^{k_{n}}+1$ for unequal powers and $k_{n}+1$ for equal powers, and the number of possibilities for $R_{\sigma}^{(s)}$ and $R_{\sigma-1}^{(s)}$ are $2^{k_{n}}-1$ each, since $R_{\sigma}^{(s)}$ and $R_{\sigma-1}^{(s)}$ cannot be empty. The above argument leads to an overall factor of $\left(2^{k_{n}}+\right.$ 1) $\left(2^{k_{n}}-1\right)^{2} \leq 8^{k_{n}}$ for unequal powers and $\left(k_{n}+1\right)\left(2^{k_{n}}-\right.$ $1)^{2} \leq\left(k_{n}+1\right) 4^{k_{n}}$ for equal powers. This completes the proof, since for unequal powers

$$
\begin{aligned}
\mathbb{P}\left(\hat{b}_{10}^{(s)} \neq b_{10}\right) & \leq \mathbb{P}\left(\bigcup _ { \sigma \leq 2 ^ { k _ { n } + 1 } } \left\{P_{\left.\left.R_{\sigma}^{(s)} \geq P_{R_{\sigma-1}^{(s)}}\right\}\right)}\right.\right. \\
& \leq 8^{k_{n}} \min _{A, B: P_{B} \geq P_{A}} \mathbb{P}\left(R_{\sigma}^{(s)}=A, R_{\sigma-1}^{(s)}=B\right) \\
& \leq 8^{k_{n}} e^{-I n} .
\end{aligned}
$$

For equal powers the proof is identical when we note that in this case $\sigma \leq k_{n}+1$. We note that the above proof also holds if we choose the $\operatorname{sign}(0)$ to be equal to \pm independently every time.

Then proof of (42) is identical to the above proof, using (51) together with the fact that whenever $s=\left\lceil\epsilon^{-1} \log \left(P / P^{\min }\right)\right\rceil+1$, there must be a $\sigma \leq\left\lceil\epsilon^{-1} \log \left(P / P^{\text {min }}\right)\right\rceil+1$ such that $P_{R_{\sigma}^{(s)}} \geq$ $(1-\epsilon) P_{R_{\sigma-1}^{(s)}}$.

\section{Proof of Theorem III.2 b)}

In order to prove Theorem III.2 b), we have to find a strategy that has asymptotic rate $\frac{1}{2} \log 2-\frac{1}{4}$. For simplicity, we will assume that all powers are equal. The proof for unequal powers is more technical and is therefore deferred to the Appendix. For simplicity, we assume that the powers are all equal to 2 . We note that when

$$
R_{2}=R_{1}
$$

that necessarily for all $\sigma \geq 1$

$$
R_{\sigma}=R_{1}
$$

Hence, we have now found a strategy that implies bit errors at all stages, so that the rate of this event is an upper bound for the rate of the optimal system. We fix $r=\left|R_{1}\right|$ and for technical reasons we assume $r$ to be odd. We will first investigate the rate

$$
-\lim _{n \rightarrow \infty} \frac{1}{n} \log \mathbb{P}\left(R_{2}=R_{1},\left|R_{1}\right|=r\right) \text {. }
$$

Due to the fact that all users are exchangeable, and the rate function of the vector $\left(\left(Z_{m}^{(1)}, Z_{m}^{(2)}\right)\right)_{m=1}^{k}$ is convex, we have that

$$
\begin{aligned}
-\lim _{n \rightarrow \infty} \frac{1}{n} \log \mathbb{P}\left(R_{2}=R_{1},\left|R_{1}\right|=r\right) \\
=-\lim _{n \rightarrow \infty} \frac{1}{n} \log \mathbb{P}\left(\sum_{m=1}^{r} Z_{m}^{(1)} \leq 0, \sum_{m=r+1}^{k} Z_{m}^{(1)} \geq 0,\right. \\
\left.\sum_{m=1}^{r} \bar{Z}_{m}^{(2)} \leq 0, \sum_{m=r+1}^{k} \bar{Z}_{m}^{(2)} \geq 0\right)
\end{aligned}
$$

where $\bar{Z}_{m}^{(2)}$ denotes $Z_{m}^{(2)}$ where the signs of the $Z_{m}^{(1)}$ are substituted. This statement will be proven in more detail in the Appendix.

We next note that

$$
\begin{aligned}
\sum_{m=1}^{k} Z_{m}^{(1)} & =\frac{1}{n} \sum_{i=1}^{n}\left(\sum_{m=1}^{k}\left(1+\sum_{\substack{j=1 \\
j \neq m}}^{k} A_{j i} A_{m i}\right)\right) \\
& =\frac{1}{n} \sum_{i=1}^{n}\left(\sum_{m, j=1}^{k} A_{j i} A_{m i}\right) \\
& =\frac{1}{n} \sum_{i=1}^{n}\left(\sum_{m=1}^{k} A_{m i}\right)^{2} \geq 0 .
\end{aligned}
$$

Moreover, we have that $\sum_{m=1}^{r} Z_{m}^{(1)} \leq 0$ for the event of interest. Hence, $\sum_{m=r+1}^{k} Z_{m}^{(1)} \geq 0$, so that we can remove the event $\left\{\sum_{m=r+1}^{k} Z_{m}^{(1)} \geq 0\right\}$. Since

$$
\mathbb{P}(X \leq 0, Y \leq 0) \geq \mathbb{P}(X-Y / 2 \leq 0, Y \leq 0)
$$

we can bound the exponential rate from above by

$$
\begin{aligned}
& -\lim _{n \rightarrow \infty} \frac{1}{n} \\
& \cdot \log \mathbb{P}\left(\sum_{m=1}^{r}\left[Z_{m}^{(1)}-\frac{1}{2} \bar{Z}_{m}^{(2)}\right] \leq 0, \sum_{m=1}^{r} \bar{Z}_{m}^{(2)} \leq 0, \sum_{m=r+1}^{k} \bar{Z}_{m}^{(2)} \geq 0\right)
\end{aligned}
$$

where for $1 \leq m \leq r$,

$$
\begin{aligned}
Z_{m}^{(1)}-\frac{1}{2} \bar{Z}_{m}^{(2)} & =\frac{1}{n} \sum_{i=1}^{n}\left(\frac{1}{2}+\sum_{j=r+1}^{k} A_{j i} A_{m i}\right) \\
\bar{Z}_{m}^{(2)} & =\frac{1}{n} \sum_{i=1}^{n}\left(1+2 \sum_{\substack{j=1 \\
j \neq m}} A_{j i} A_{m i}\right) \\
& =\frac{1}{n} \sum_{i=1}^{n}\left(-1+2 \sum_{j=1}^{r} A_{j i} A_{m i}\right)
\end{aligned}
$$

while for $r+1 \leq m \leq k$

$$
\bar{Z}_{m}^{(2)}=\frac{1}{n} \sum_{i=1}^{n}\left(1+2 \sum_{j=1}^{r} A_{m i} A_{j i}\right) .
$$

We abbreviate

$$
E_{1}=\left\{-r+2 \sum_{m, j=1}^{r} \frac{1}{n} \sum_{i=1}^{n} A_{m i} A_{j i} \leq 0\right\}
$$




$$
\begin{aligned}
& E_{2}=\left\{\frac{r}{2}+\sum_{m=r+1}^{k} \sum_{j=1}^{r} \frac{1}{n} \sum_{i=1}^{n} A_{m i} A_{j i} \leq 0\right\} \\
& E_{3}=\left\{\sum_{m=r+1}^{k} \sum_{j=1}^{r}\left[1+\frac{1}{n} \sum_{i=1}^{n} A_{m i} A_{j i}\right] \geq 0\right\} .
\end{aligned}
$$

Clearly

$$
\mathbb{P}\left(E_{1} \cap E_{2}\right)=\mathbb{P}\left(E_{1} \cap E_{2} \cap E_{3}\right)+\mathbb{P}\left(E_{1} \cap E_{2} \cap E_{3}^{c}\right)
$$

so that, according to the largest exponent wins principle

$$
\begin{aligned}
-\lim _{n \rightarrow \infty} \frac{1}{n} \log \mathbb{P}\left(E_{1} \cap E_{2}\right)= & \min \left\{-\lim _{n \rightarrow \infty} \frac{1}{n} \log \mathbb{P}\left(E_{1} \cap E_{2} \cap E_{3}\right),\right. \\
& \left.-\lim _{n \rightarrow \infty} \frac{1}{n} \log \mathbb{P}\left(E_{1} \cap E_{2} \cap E_{3}^{c}\right)\right\} .
\end{aligned}
$$

We wish to show that for $k \rightarrow \infty$ and $r$ large

- $\lim _{n \rightarrow \infty} \frac{1}{n} \log \mathbb{P}\left(E_{1} \cap E_{2}\right)=-\lim _{n \rightarrow \infty} \frac{1}{n} \log \mathbb{P}\left(E_{1} \cap E_{2} \cap E_{3}\right)$. In order to do so, we show that

$$
\liminf _{k \rightarrow \infty}-\lim _{n \rightarrow \infty} \frac{1}{n} \log \mathbb{P}\left(E_{1} \cap E_{2} \cap E_{3}^{c}\right) \geq \frac{1}{2} \log \frac{3}{4}+\frac{1}{4} .
$$

for all $r$. Furthermore, we will show that

$$
\text { - } \lim _{k \rightarrow \infty} \lim _{n \rightarrow \infty} \frac{1}{n} \log \mathbb{P}\left(E_{1} \cap E_{2}\right) \leq-\lim _{n \rightarrow \infty} \frac{1}{n} \log \mathbb{P}\left(E_{1}\right)
$$
and

$$
-\lim _{n \rightarrow \infty} \frac{1}{n} \log \mathbb{P}\left(E_{1}\right)=\frac{1}{2} \log 2-\frac{1}{4}+o(1)
$$

as $r \rightarrow \infty$. Because the three statements $u \leq \min \{v, w\}, u \leq$ $x$, and $w>x$ imply $u \leq x$, this implies directly when $r$ is large

$$
\text { - } \lim _{k \rightarrow \infty} \lim _{n \rightarrow \infty} \frac{1}{n} \log \mathbb{P}\left(E_{1} \cap E_{2} \cap E_{3}\right) \frac{1}{2} \log 2-\frac{1}{4}+o(1) .
$$

Equations (69)-(71) also imply the statement in Theorem III. 2 b). Indeed, taking $r \rightarrow \infty$ gives

$$
-\lim _{r \rightarrow \infty} \lim _{k \rightarrow \infty} \lim _{n \rightarrow \infty} \frac{1}{n} \log \mathbb{P}\left(E_{1} \cap E_{2} \cap E_{3}\right) \leq \frac{1}{2} \log 2-\frac{1}{4} .
$$

The remainder of this proof, therefore, focuses on proving (69)-(71). We prove (69) in the following lemma, (70) in Lemma V.3, and (71) in Lemma V.4.

Lemma V.2: As $k \rightarrow \infty$

$\liminf _{k \rightarrow \infty}-\lim _{n \rightarrow \infty} \frac{1}{n} \log \mathbb{P}\left(E_{1} \cap E_{2} \cap E_{3}^{c}\right) \geq \frac{1}{2} \log \frac{3}{4}+\frac{1}{4}$.

Proof: We bound the rate of interest from below by

$-\lim _{n \rightarrow \infty} \frac{1}{n} \log \mathbb{P}\left(E_{3}^{c}\right)$

$=-\lim _{n \rightarrow \infty} \frac{1}{n} \log \mathbb{P}\left(\sum_{m=r+1}^{k} \bar{Z}_{m}^{(2)} \leq 0\right)$

$=-\lim _{n \rightarrow \infty} \frac{1}{n} \log \mathbb{P}\left(\frac{1}{n} \sum_{i=1}^{n} \sum_{m=r+1}^{k}\left[1+2 \sum_{j=1}^{r} A_{j i} A_{m i}\right]<0\right)$.

We can follow the proof of Section V-A with $A=\{0, \ldots, r-1\}$ and $B=\{r, \ldots, k-1\}$ (see, e.g., (60) with $A \cap B=\varnothing$ ). This results in

$$
-\lim _{n \rightarrow \infty} \frac{1}{n} \log \mathbb{P}\left(E_{3}^{c}\right) \geq \frac{1}{2} \log \left(1-\frac{r}{4(k-r)}\right)+\frac{1}{4}
$$

which exceeds $\frac{1}{2} \log \frac{3}{4}+\frac{1}{4}$ when $r \leq k / 2$.
The strategy of the proof is first to characterize the behavior for $k \rightarrow \infty$ and then showing that $r \rightarrow \infty$ gives the desired result.

Lemma V.3: For $r$ fixed

$\lim _{k \rightarrow \infty}-\lim _{n \rightarrow \infty} \frac{1}{n} \log \mathbb{P}\left(E_{1} \cap E_{2}\right) \leq-\lim _{n \rightarrow \infty} \frac{1}{n} \log \mathbb{P}\left(E_{1}\right)$.

Proof: We focus on

$$
\begin{aligned}
-\lim _{n \rightarrow \infty} \frac{1}{n} \log \mathbb{P}\left(\frac{r}{2}+\sum_{m=r}^{k-1}\right. & \sum_{j=1}^{r} \frac{1}{n} \sum_{i=1}^{n} A_{m i} A_{j i} \leq 0, \\
-r & \left.+2 \sum_{m, j=1}^{r} \frac{1}{n} \sum_{i=1}^{n} A_{m i} A_{j i} \leq 0\right) .
\end{aligned}
$$

Using Cramér's theorem and invoking the notation

$$
R=\{0, \ldots, r-1\} \quad \text { and } R_{0}^{+}=\{r, \ldots, k-1\}
$$

gives that the rate above is given by

$$
-\inf _{t_{1}, t_{2} \leq 0} \log \mathbb{E}\left(e^{t_{1}\left(r / 2+S_{R} S_{R_{0}^{+}}\right) t_{2}\left(2 S_{R}^{2}-r\right)}\right) .
$$

Since $S_{R}$ and $S_{R_{0}^{+}}$are independent, we can bound the rate of interest from above by

$$
-\inf _{t_{1}, t_{2} \leq 0} \log \left(e^{t_{1} r / 2} \cosh \left(t_{1} S_{R}\right)^{k-r} \mathbb{E} e^{t_{2}\left(2 S_{R}^{2}-r\right)}\right) .
$$

It is sufficient to prove that for all $\delta>0,\left|t_{1}\right| \leq \delta$ as $k \rightarrow \infty$. Indeed, since $\cosh x \geq 1$, the rate on the right-hand side is bounding (74) from above by

$$
\begin{aligned}
- & \inf _{\left|t_{1}\right| \leq \delta, t_{2} \leq 0} \log \left(e^{t_{1} r / 2}\left(\cosh \left(t_{1} S_{R}\right)\right)^{k-r} \mathbb{E} e^{t\left(2 S_{R}^{2}-r\right)}\right) \\
& \leq-\inf _{t_{2} \leq 0} \log \left(e^{-\delta r / 2} \mathbb{E} e^{t\left(2 S_{R}^{2}-r\right)}\right) \\
& =\frac{\delta r}{2}-\inf _{t_{2}} \log \mathbb{E} e^{t_{2}\left(2 S_{R}^{2}-r\right)}=\frac{\delta r}{2}-\lim _{n \rightarrow \infty} \frac{1}{n} \log \mathbb{P}\left(E_{1}\right) .
\end{aligned}
$$

Since $\delta$ is arbitrarily small and $r$ is fixed, the desired statement then follows. It is hence sufficient to prove that under $\left|t_{1}\right| \geq \delta$, the infimum cannot be attained for $k$ sufficiently large. We prove this statement by contradiction. Assume that the infimum is attained for a $\left|t_{1}\right| \geq \delta$. Since $r$ is odd, $\cosh \left(t_{1} j\right) \geq \cosh \left(t_{1}\right) \geq$ $e^{\left|t_{1}\right|} / 2$, so that

$$
e^{t_{1} r / 2} \cosh \left(t_{1} j\right)^{r} \geq 2^{-r} e^{-\left|t_{1}\right| r / 2} e^{r\left|t_{1}\right|} \geq 2^{-r} .
$$

Substituting the above result gives

$$
\begin{aligned}
& -\inf _{\left|t_{1}\right| \geq \delta, t_{2} \leq 0} \log \left(e^{t_{1} r / 2}\left(\cosh \left(t_{1} j\right)\right)^{k-r} \mathbb{E} e^{t\left(2 S_{R}^{2}-r\right)}\right) \\
& \leq-\inf _{\left|t_{1}\right| \geq \delta, t_{2} \leq 0} \log \left(e^{-r \log 2}\left(\cosh \left(t_{1} j\right)\right)^{k-2 r} \mathbb{E} e^{t\left(2 S_{R}^{2}-r\right)}\right) \\
& \leq-\inf _{t_{2} \leq 0} \log \left(e^{-r \log 2}(\cosh \delta)^{k-2 r} \mathbb{E} e^{t\left(2 S_{R}^{2}-r\right)}\right) \\
& =-\inf _{t_{2} \leq 0} \log \mathbb{E} e^{t_{2}\left(2 S_{R}^{2}-r\right)}+r \log 2-(k-2 r) \log \cosh \delta .
\end{aligned}
$$

Since $\cosh (\delta)>1$, the last term tends to $-\infty$ for $k \rightarrow \infty$. Thus, assuming $t_{1} \geq \delta$, it leads to $-\lim _{n \rightarrow \infty} \frac{1}{n} \log \mathbb{P}\left(E_{1}\right)<0$, which is a contradiction.

To complete the proof, we let $r \rightarrow \infty$.

Lemma V.4: For $r \rightarrow \infty$

$$
\text { - } \lim _{n \rightarrow \infty} \frac{1}{n} \log \mathbb{P}\left(E_{1}\right) \leq \frac{1}{2} \log 2-\frac{1}{4}+o(1) .
$$


Proof: We show

$$
\lim _{r \rightarrow \infty}-\inf _{t_{2} \leq 0} \log \mathbb{E} e^{t_{2}\left(2 S_{r}^{2}-r\right)}=\frac{1}{2} \log 2-\frac{1}{4}
$$

The first step is to show that whenever $t_{2} \leq-1 / r, h\left(t_{2}\right)=$ $\mathbb{E} e^{t_{2}\left(2 S_{r}^{2}-r\right)}>1$. We then can conclude that the infimum is not attained, since $h(0)=1<h\left(t_{2}\right)$ for $t_{2} \leq-1 / r$. Since $h\left(t_{2}\right)$ is a moment-generating function, it is log-convex, so that it suffices to show that $h(-1 / r)>1$. Indeed, for all $t_{2} \leq-1 / r$, there exists an $\alpha \in(0,1]$, such that $\alpha t_{2}=-1 / r$. It now follows that

$$
\begin{aligned}
1<h\left(\alpha t_{2}\right) & =h\left(\alpha t_{2}+(1-\alpha) \cdot 0\right) \\
& \leq \alpha h\left(t_{2}\right)+(1-\alpha) h(0)=\alpha h\left(t_{2}\right)+(1-\alpha)
\end{aligned}
$$

so that $h\left(t_{2}\right)>1$. In order to prove that $h(-1 / r)>1$, we observe that

$$
e^{\left(-\frac{1}{r}\right)\left(2 S_{r}^{2}-r\right)}=e^{1-2\left(\frac{S_{r}}{\sqrt{r}}\right)^{2}} \stackrel{\mathcal{D}}{\rightarrow} e^{1-2 Z^{2}}
$$

where $Z \stackrel{d}{=} \mathcal{N}(0,1)$. Furthermore, since $\mathbb{E} e^{\left(-\frac{\alpha}{r}\right)\left(2 S_{r}^{2}-r\right)} \leq e^{\alpha}<$ $\infty$ for all $\alpha>1$, it then follows from [7, Example 7.10.15] that as $r \rightarrow \infty$

$$
\mathbb{E} e^{1-2\left(\frac{S_{r}}{\sqrt{r}}\right)^{2}} \rightarrow \mathbb{E} e^{1-2 Z^{2}}=\frac{e}{\sqrt{5}} \approx 1.21565>1 .
$$

Indeed, the infimum is not attained. Finally, using that $t_{2}=$ $-\beta / r$ for some $\beta \in[0,1)$, we can again use the argument above to conclude that

$$
\mathbb{E} e^{\beta-2 \beta\left(\frac{S_{r}}{\sqrt{r}}\right)^{2}} \rightarrow \mathbb{E} e^{\beta-2 \beta Z^{2}}=\frac{e^{\beta}}{\sqrt{1+4 \beta}} .
$$

Minimizing over $\beta$ gives $\beta^{*}=1 / 4$, resulting in

$$
\lim _{r \rightarrow \infty}-\inf _{t_{2} \leq 0} \log \mathbb{E} e^{t_{2}\left(2 S_{r}^{2}-r\right)}=-\log \frac{e^{1 / 4}}{\sqrt{2}}=\frac{1}{2} \log 2-\frac{1}{4}
$$

which is the desired result.

This completes the proof of Theorem III.2 b) in the case of equal powers.

\section{CONCLUSION}

We Investigated a DS-CDMA system with HD-PIC. The model incorporates interfering users with unequal powers. Large deviation theory is used to obtain qualitative statements concerning the performance of the system when the number of users is small compared to the processing gain. The exponential rate has been proven to be a good measure of performance. For the MF system, the exponential rate is asymptotically equivalent to the well-known SNR. We note that for a lightly loaded HD-PIC system the SNR is not a good measure of performance, since there is no Gaussian behavior for large $n$. However, the exponential rate of the BEP is not based on Gaussian assumptions and this allowed us to draw qualitative conclusions concerning the behavior of HD-PIC systems by using the exponential rate of the BEP. The large deviation results often gives useful starting points for the system in which the number of users is comparable with the processing gain.

We also investigated the asymptotic exponential rate of the BEP for multistage HD-PIC as the number of users tends to infinity. The asymptotic exponential rate of the BEP showed that HD-PIC keeps increasing performance as the number of users increases.
We showed that the exponential rate of the BEP remains unchanged after a finite number of HD-PIC stages. The number of stages of HD-PIC after which this happens is one for three to nine users, and two for 10 up to 22 users when all powers are equal. This yields the first analytical proof of this effect often observed in practice. We have shown that the exponential rate of the BEP remains uniformly positive as the number of users increases. Under mild conditions on the powers, we identified the limiting exponential rate of the BEP after applying sufficiently stages of HD-PIC as $\frac{1}{2} \log 2-\frac{1}{4}$ when the number of users increases.

The exponential rate was used in a Chernoff bound. The bound is not tight in the sense that it approximates the BEP with any desired precision. Instead, it gives an upper bound that is valid for all $n$ and $k$, making it a robust upper bound. For $s$ fixed, the Chernoff bound is valid as long as $k=o\left(\frac{\log n}{n}\right)$, whereas for the optimal HD-PIC system, it is valid as long as $k=o(n)$, and even when $k \leq \delta n$ for some positive $\delta$. For the case

$$
s \geq\left\lceil\epsilon^{-1} \log \frac{P}{P^{\min }}\right\rceil+1, \quad \text { with } \epsilon<\frac{1}{2} \log 2-\frac{1}{4}
$$

and $P / P^{\text {min }}$ is the ratio of the total power and the minimum power, we identified the lower bound of the exponential rate as a function of $\epsilon$. Furthermore, we showed that for both the optimal system and the system in which $s$ is sufficiently large, the BEP tends to zero exponentially fast, even when $k \leq \delta n$ for some positive $\delta$.

The analysis in this paper answered a number of questions. Nevertheless, many other questions remain open. We try to summarize the most important open questions.

1) What happens to the results when AWGN and unequal powers are considered in the case where we apply more than two stages of HD-PIC? We expect that the result will be generalized as in (25), even though the conditions on the powers are likely to be more involved.

2) Can we include AWGN in the proof for the optimal HD-PIC system? We believe that we can prove upper and lower bounds on the asymptotic exponential rate of the BEP using the methods in this paper, but that these bounds are not sharp. In order to prove asymptotics, new ideas will need to be developed.

3) Can we say more about how many iterations of HD-PIC are necessary to obtain the optimal system? For instance, can we prove Conjecture III.6 ?

4) When is the BEP exponentially small for the optimal HD-PIC system and $k_{n}=\delta n$ ? Is this true for all $\delta>0$ or not, i.e., is there a phase transition for some positive $\delta^{*}$ such that the BEP is exponentially small for $\delta<\delta^{*}$, whereas it is not exponentially small for $\delta>\delta^{*}$ ?

\section{APPENDIX}

\section{PROOF OF THEOREM III.2 b) FOR UNEQUAL POWERS}

We recall that we have the following condition on the powers $\left(P_{1}, \ldots, P_{k}\right)$ :

$\left(\mathcal{P}_{1}\right)$ there exists a $\delta>0$ s. t. $\sharp\left\{j: P_{j} \in[\delta, 1 / \delta]\right\} \rightarrow \infty$; 
$\left(\mathcal{P}_{2}\right) \lim _{k \rightarrow \infty} k P^{-1}<\infty$

$\left(\mathcal{P}_{3}\right) k^{-1} P^{\max } \rightarrow 0$

$\left(\mathcal{P}_{4}\right) k P^{\min } \rightarrow \infty$;

where $P^{\max }=\max _{m} P_{m}, P^{\min }=\min _{m} P_{m}$. We further denote by $R^{c}$ the complement of $R$, defined as $\{1, \ldots, k\} \backslash R$.

The result of the theorem is that under these power conditions, we have that

$$
\lim _{k \rightarrow \infty} H_{k}^{\left(s_{k}\right)}=\frac{1}{2} \log 2-\frac{1}{4} .
$$

Theorem III.2 a) states that $H_{k}^{\left(s_{k}\right)} \geq \frac{1}{2} \log 2-\frac{1}{4}$, so that it is sufficient to prove

$$
\lim _{k \rightarrow \infty} H_{k}^{\left(s_{k}\right)} \leq \frac{1}{2} \log 2-\frac{1}{4} .
$$

Similarly to the case in which all powers are equal, we will focus on the event $\left\{R_{2}=R_{1}=R\right\}$. This specifies $R_{\sigma}$ for all $\sigma$. We will show the theorem, using a symmetrizing argument. More precisely, we replace different powers by the same value. In this case, we can use exchangeability, together with convexity arguments in order to simplify the analysis. We intend to follow the strategy in Section V-C as closely as possible.

We take $R \subset\left\{j: P_{j} \in[\delta, 1 / \delta]\right\}$ and we assume that $|R|$ is fixed and odd and $k$ is large.

Our starting point is the probability

$$
\begin{aligned}
\mathbb{P}\left(R_{2}=R_{1}=R\right)=\mathbb{P}\left(\max _{m \in R} Z_{m}^{(1)} \leq 0, \min _{m \in R^{c}} Z_{m}^{(1)} \geq 0,\right. \\
\left.\max _{m \in R} \bar{Z}_{m}^{(2)} \leq 0, \min _{m \in R^{c}} \bar{Z}_{m}^{(2)} \geq 0\right)
\end{aligned}
$$

where

$$
\begin{aligned}
& Z_{m}^{(1)}=\frac{1}{n} \sum_{i=1}^{n}\left(P_{m}^{1 / 2}+\sum_{\substack{j=1 \\
j \neq m}}^{k} P_{j}^{1 / 2} A_{j i} A_{m i}\right) \\
& \bar{Z}_{m}^{(2)}=\frac{1}{n} \sum_{i=1}^{n}\left(P_{m}^{1 / 2}+2 \sum_{\substack{j \in R \\
j \neq m}} P_{j}^{1 / 2} A_{j i} A_{m i}\right) .
\end{aligned}
$$

Since $\mathbb{P}(A \leq 0, B \leq 0) \geq \mathbb{P}(A-B / 2 \leq 0, B \leq 0)$, we can bound the probability from below by

$$
\begin{aligned}
\mathbb{P}\left(\max _{m \in R} Z_{m}^{(1)}-\frac{1}{2} \bar{Z}_{m}^{(2)} \leq 0,\right. & \min _{m \in R^{c}} Z_{m}^{(1)}-\frac{1}{2} \bar{Z}_{m}^{(2)} \geq 0, \\
& \left.\max _{m \in R} \bar{Z}_{m}^{(2)} \leq 0, \min _{m \in R^{c}} \bar{Z}_{m}^{(2)} \geq 0\right)
\end{aligned}
$$

where for $m \in R$

$$
\begin{aligned}
Z_{m}^{(1)}-\frac{1}{2} \bar{Z}_{m}^{(2)} & =\frac{1}{n} \sum_{i=1}^{n}\left(\frac{1}{2} P_{m}^{1 / 2}+\sum_{j \in R^{c}} P_{j}^{1 / 2} A_{j i} A_{m i}\right) \\
\bar{Z}_{m}^{(2)} & =\frac{1}{n} \sum_{i=1}^{n}\left(P_{m}^{1 / 2}+2 \sum_{\substack{j \in R \\
j \neq m}} P_{j}^{1 / 2} A_{j i} A_{m i}\right)
\end{aligned}
$$

while for $m \in R^{c}$

$$
\begin{aligned}
Z_{m}^{(1)}-\frac{1}{2} \bar{Z}_{m}^{(2)} & =\frac{1}{n} \sum_{i=1}^{n}\left(\frac{1}{2} P_{m}^{1 / 2}+\sum_{\substack{j \in R^{c} \\
j \neq m}} P_{j}^{1 / 2} A_{j i} A_{m i}\right) \\
\bar{Z}_{m}^{(2)} & =\frac{1}{n} \sum_{i=1}^{n}\left(P_{m}^{1 / 2}+2 \sum_{j \in R} P_{j}^{1 / 2} A_{j i} A_{m i}\right) .
\end{aligned}
$$

We can further bound the probability of interest from below by the probability of the intersection of the events

$$
\begin{aligned}
\max _{m \in R, j \in R^{c}} \frac{1}{n} \sum_{i=1}^{n} A_{m i} A_{j i} & \leq-\frac{\left(P_{R^{c}}^{\max } P_{R}^{\max }\right)^{1 / 2}}{2 P_{R^{c}}} \\
\max _{m, j \in R, j \neq m} \frac{1}{n} \sum_{i=1}^{n} A_{m i} A_{j i} & \leq-\frac{\left(P_{R}^{\max }\right)^{1 / 2}}{2(|R|-1)\left(P_{R}^{\min }\right)^{1 / 2}} \\
\min _{m, j \in R^{c}, j \neq m} \frac{1}{n} \sum_{i=1}^{n} A_{m i} A_{j i} & \geq 0 \\
\min _{m \in R, j \in R^{c}} \frac{1}{n} \sum_{i=1}^{n} A_{m i} A_{j i} & \geq-\frac{\left(P_{R^{c}}^{\min }\right)^{1 / 2}}{2|R|\left(P_{R}^{\max }\right)^{1 / 2}}
\end{aligned}
$$

where $P_{R}^{\max }=\max _{m \in R} P_{m}$ and $P_{R}^{\min }=\min _{m \in R} P_{m}$.

Indeed, using

we have for $m \in R$

$$
\sum_{m \in R^{c}} P_{m}^{1 / 2} \geq \frac{P_{R^{c}}}{\left(P_{R^{c}}^{\max }\right)^{1 / 2}}
$$

$$
\begin{aligned}
Z_{m}^{(1)}-\frac{\bar{Z}_{m}^{(2)}}{2} & =\frac{1}{n} \sum_{i=1}^{n}\left(\frac{1}{2} P_{m}^{1 / 2}+\sum_{j \in R^{c}} P_{j}^{1 / 2} A_{j i} A_{m i}\right) \\
& \leq \frac{1}{2}\left(P_{R}^{\max }\right)^{1 / 2}-\sum_{j \in R^{c}} P_{j}^{1 / 2} \frac{\left(P_{R^{c}}^{\max } P_{R}^{\max }\right)^{1 / 2}}{2 P_{R^{c}}} \\
& \leq \frac{1}{2}\left(P_{R}^{\max }\right)^{1 / 2}-\frac{\left(P_{R}^{\max }\right)^{1 / 2}}{2}=0 .
\end{aligned}
$$

Furthermore

$$
\begin{aligned}
\bar{Z}_{m}^{(2)} & =\frac{1}{n} \sum_{i=1}^{n}\left(P_{m}^{1 / 2}+2 \sum_{\substack{j \in R \\
j \neq m}} P_{j}^{1 / 2} A_{j i} A_{m i}\right) \\
& \leq\left(P_{R}^{\max }\right)^{1 / 2}-2 \sum_{\substack{j \in R \\
j \neq m}}\left(P_{R}^{\min }\right)^{1 / 2} \frac{\left(P_{R}^{\max }\right)^{1 / 2}}{2(|R|-1)\left(P_{R}^{\min }\right)^{1 / 2}} \\
& \leq\left(P_{R}^{\max }\right)^{1 / 2}-\frac{(|R|-1)\left(P_{R}^{\max } P_{R}^{\min }\right)^{1 / 2}}{(|R|-1)\left(P_{R}^{\min }\right)^{1 / 2}}=0 .
\end{aligned}
$$

For $m \in R^{c}$, similar arguments give the desired result

$$
\begin{aligned}
Z_{m}^{(1)}-\frac{\bar{Z}_{m}^{(2)}}{2}= & \frac{1}{n} \sum_{i=1}^{n}\left(\frac{1}{2} P_{m}^{1 / 2}+\sum_{j \in R^{c}} P_{j}^{1 / 2} A_{j i} A_{m i}\right) \\
\geq & \frac{1}{2} P_{m}^{1 / 2} \geq 0, \\
\bar{Z}_{m}^{(2)}= & \frac{1}{n} \sum_{i=1}^{n}\left(P_{m}^{1 / 2}+2 \sum_{j \in R} P_{j}^{1 / 2} A_{j i} A_{m i}\right) \\
\geq & \left(P_{R^{c}}^{\min }\right)^{1 / 2}-2 \\
& \cdot \sum_{j \in R}\left(P^{\max }\right)^{1 / 2} \frac{\left(P_{R^{c}}^{\min }\right)^{1 / 2}}{2|R|\left(P^{\max } R\right)^{1 / 2}} \\
= & 0 .
\end{aligned}
$$


In order to obtain a positive probability, we must assure that

$$
-\frac{\left(P^{\max } R^{c} P^{\max }{ }^{1 / 2}\right.}{2 P_{R^{c}}} \geq-\frac{\left(P_{R^{c}}^{\min }\right)^{1 / 2}}{2|R|\left(P^{\max } R\right)^{1 / 2}}
$$

or equivalently

$$
\frac{\left(P^{\max }\right)^{2} P^{\max } R^{c}|R|^{2}}{P_{R^{c}}^{\min } P_{R^{c}}^{2}} \leq 1
$$

We use $P^{\max } R^{c} \leq P^{\text {max }}$, together with $P_{R^{c}}^{\min } \geq P^{\text {min }}$ and $P^{\max }{ }_{R} \leq 1 / \delta$, to obtain from $\left(\mathcal{P}_{2}\right),\left(\mathcal{P}_{3}\right)$, and $\left(\mathcal{P}_{4}\right)$ that

$$
\begin{aligned}
\frac{\left(P^{\max }{ }_{R}\right)^{2} P^{\max } R_{R^{c}}|R|^{2}}{P_{R^{c}}^{\min } P_{R^{c}}^{2}} & \leq \delta^{-2}|R|^{2} \frac{P^{\max }}{P^{2} P^{\min }} \\
& =\delta^{-2}|R|^{2} \frac{k^{2}}{P^{2}} \frac{P^{\max }}{k} \frac{1}{k P^{\min }}
\end{aligned}
$$

which tends to zero for $k \rightarrow \infty$. Hence, the inequality holds, since $|R|$ is fixed and $k$ is sufficiently large.

We have now symmetrized the problem. The next step is to observe that the events are exchangeable for $m, j$. Together with convexity of the rate function, we can replace the max and min by $\sum$, as we will now show. This is a standard large deviation argument.

We write $\vec{a} \mapsto J_{k}(\vec{a})$ to be the rate function of the cross correlations $\vec{W}=\left(W_{j m}\right)_{1 \leq j<m \leq k}$, where

$$
W_{j m}=\frac{1}{n} \sum_{i=1}^{n} A_{i j} A_{i m} .
$$

This means that, for any set $A$, we let

$$
J_{k}(A)=-\lim _{n \rightarrow \infty} \frac{1}{n} \log \mathbb{P}(\vec{W} \in A)
$$

and we can compute $J_{k}(A)$ using

$$
J_{k}(A)=\min _{\vec{a} \in A} J_{k}(\vec{a}) .
$$

This rate function $\vec{a} \mapsto J_{k}(\vec{a})$ exists by Cramér's theorem, is convex by [10, Theorem III.27], and is minimal for $\vec{a}=\overrightarrow{0}$, where it takes the value $J_{k}(\overrightarrow{0})=0$. We note that

$$
H_{k}^{\left(s_{k}\right)} \leq \min _{\vec{a} \in A} J_{k}(\vec{a})
$$

where $A$ is defined to be the set of vectors such that the coordinates satisfy the inequalities in (77). We now let

$$
\begin{aligned}
& E_{1}=\left\{\sum_{\substack{m, j \in R \\
m \neq j}} \frac{1}{n} \sum_{i=1}^{n} A_{m i} A_{j i} \leq-|R| \frac{\left(P_{R}^{\max }\right)^{1 / 2}}{2\left(P_{R}^{\min }\right)^{1 / 2}}\right\} \\
& E_{2}=\left\{\sum_{\substack{m \in R \\
j \in R^{c}}} \frac{1}{n} \sum_{i=1}^{n} A_{m i} A_{j i} l e q-|R|\left|R^{c}\right| \frac{\left(P_{R^{c}}^{\max } P_{R}^{\max }\right)^{1 / 2}}{2 P_{R^{c}}}\right\} \\
& E_{3}=\left\{\sum_{\substack{m, j \in R^{c} \\
m \neq j}} \frac{1}{n} \sum_{i=1}^{n} A_{m i} A_{j i} \geq 0\right\} \\
& E_{4}=\left\{\begin{array}{c}
\left.\sum_{\substack{m \in R \\
j \in R^{c}}} \frac{1}{n} \sum_{i=1}^{n} A_{m i} A_{j i} \geq-|R|\left|R^{c}\right| \frac{\left(P_{R^{c}}^{\min }\right)^{1 / 2}}{2|R|\left(P_{R}^{\max }\right)^{1 / 2}}\right\} .(83)
\end{array}\right.
\end{aligned}
$$

Then we see that

$$
\text { - } \lim _{n \rightarrow \infty} \frac{1}{n} \log \mathbb{P}\left(E_{1} \cap E_{2} \cap E_{3} \cap E_{4}\right)=\min _{\vec{b} \in B} J_{k}(\vec{b})
$$

where $B$ is the set such that the inequalities in (83) hold. Note that $A \subset B$, and we wish to show that the minimum in (84) is attained for a $\vec{b}^{*} \in A$. That proves that the rate on the right-hand side of (82) equals the rate of $\mathbb{P}\left(E_{1} \cap E_{2} \cap E_{3} \cap E_{4}\right)$.

Let $\vec{b}^{*}$ be the minimizer of (84). Note that by exchangeability, if we interchange the elements in $R$ and $R^{c}$, we obtain the same rate. We write $\mathcal{P}_{R}$ for the set of permutations of the elements in $R$ and $\mathcal{P}_{R^{c}}$, the set of permutations of the elements in $R^{c}$, and we define $\mathcal{P}=\mathcal{P}_{R} \times \mathcal{P}_{R^{c}}$. Hence, for a $p \in \mathcal{P}$, we have $p=\left(p_{1}, p_{2}\right)$ where $p_{1} \in \mathcal{P}_{R}, p_{2} \in \mathcal{P}_{R^{c}}$.

Then we obtain that

$$
\min _{\vec{b} \in B} J_{k}(\vec{b})=\frac{1}{|\mathcal{P}|} \sum_{p \in \mathcal{P}} J_{k}\left(p\left(\vec{b}^{*}\right)\right) .
$$

By convexity, we obtain that

$$
\min _{\vec{b} \in B} J_{k}(\vec{b}) \geq J_{k}\left(\frac{1}{|\mathcal{P}|} \sum_{p \in \mathcal{P}} p\left(\vec{b}^{*}\right)\right) .
$$

We end up with the vector $\vec{a}^{*}=\frac{1}{|\mathcal{P}|} \sum_{p \in \mathcal{P}} p\left(\vec{b}^{*}\right)$. This vector takes only three different values, as we will explain now. Indeed, let $j \neq j^{\prime}$ be any two indexes in $R$, and $m \neq m^{\prime}$ any two indexes in $R^{c}$. Then $a_{n l}^{*}$ takes the value $a_{j j^{\prime}}^{*}$ for $n, l \in R$, the value $a_{j m}^{*}$ for $n \in R, l \in R^{c}$, and $a_{m m^{\prime}}^{*}$ for any $n, l \in R^{c}$. Hence, we must have that each of the elements satisfies the inequalities in (77), so that $\vec{a}^{*} \in A$. This completes the proof.

We continue to determine the rate of $\mathbb{P}\left(E_{1} \cap E_{2} \cap E_{3} \cap E_{4}\right)$. We write

$$
\begin{aligned}
\mathbb{P}\left(E_{1} \cap E_{2} \cap E_{3}\right) & \\
& =\mathbb{P}\left(E_{1} \cap E_{2} \cap E_{3} \cap E_{4}\right)+\mathbb{P}\left(E_{1} \cap E_{2} \cap E_{3} \cap E_{4}^{c}\right) .
\end{aligned}
$$

By the largest exponent wins principle, we have

$$
\begin{aligned}
-\lim _{n \rightarrow \infty} \frac{1}{n} \log \mathbb{P} & \left(E_{1} \cap E_{2} \cap E_{3}\right) \\
= & \min \left\{-\lim _{n \rightarrow \infty} \frac{1}{n} \log \mathbb{P}\left(E_{1} \cap E_{2} \cap E_{3} \cap E_{4}\right),\right. \\
& \left.\quad-\lim _{n \rightarrow \infty} \frac{1}{n} \log \mathbb{P}\left(E_{1} \cap E_{2} \cap E_{3} \cap E_{4}^{c}\right)\right\} .
\end{aligned}
$$

We wish to show that for $k \rightarrow \infty$ and $|R|$ large

$$
\begin{aligned}
-\lim _{n \rightarrow \infty} \frac{1}{n} \log \mathbb{P} & \left(E_{1} \cap E_{2} \cap E_{3}\right) \\
& =-\lim _{n \rightarrow \infty} \frac{1}{n} \log \mathbb{P}\left(E_{1} \cap E_{2} \cap E_{3} \cap E_{4}\right) .
\end{aligned}
$$

In order to do so, we show that

$$
\liminf _{k \rightarrow \infty}-\lim _{n \rightarrow \infty} \frac{1}{n} \log \mathbb{P}\left(E_{1} \cap E_{2} \cap E_{3} \cap E_{4}^{c}\right) \geq \frac{1}{2} \log \frac{3}{4}+\frac{1}{4}
$$

for all $R$. Furthermore, we will show that

$$
\text { - } \lim _{k \rightarrow \infty} \lim _{n \rightarrow \infty} \frac{1}{n} \log \mathbb{P}\left(E_{1} \cap E_{2} \cap E_{3}\right)=-\lim _{n \rightarrow \infty} \frac{1}{n} \log \mathbb{P}\left(E_{1}\right)
$$

and

$$
-\lim _{n \rightarrow \infty} \frac{1}{n} \log \mathbb{P}\left(E_{1}\right) \leq \frac{1}{2} \log 2-\frac{1}{4}+o(1)
$$


for $|R|$ sufficiently large. Because the three statements $u=$ $\min \{v, w\}, u \leq x$ and $w>x$ imply $u \leq x$, this implies directly that when $|R|$ is large

- $\lim _{k \rightarrow \infty} \lim _{n \rightarrow \infty} \frac{1}{n} \log \mathbb{P}\left(E_{1} \cap E_{2} \cap E_{3} \cap E_{4}\right) \leq \frac{1}{2} \log 2-\frac{1}{4}+o(1)$.

This prove the theorem, since indeed

$x=-\lim _{|R| \rightarrow \infty} \lim _{k \rightarrow \infty} \lim _{n \rightarrow \infty} \frac{1}{n} \log \mathbb{P}\left(E_{1} \cap E_{2} \cap E_{3}\right) \leq \frac{1}{2} \log 2-\frac{1}{4}$.

The remainder of this proof therefore focuses on proving (88)(90). We prove (88) in the following lemma, (89) in Lemma A.2, and (90) in Lemma A.3.

Lemma A.1: For $k$ sufficiently large, under power conditions $\left(\mathcal{P}_{1}\right)$ and $\left(\mathcal{P}_{4}\right)$

$-\lim _{n \rightarrow \infty} \frac{1}{n} \log \mathbb{P}\left(E_{1} \cap E_{2} \cap E_{3} \cap E_{4}^{c}\right) \geq \frac{1}{2} \log \frac{3}{4}+\frac{1}{4}$.

Proof: We have

$$
\begin{aligned}
& \text { - } \lim _{n \rightarrow \infty} \frac{1}{n} \log \mathbb{P}\left(E_{1} \cap E_{2} \cap E_{3} \cap E_{4}^{c}\right) \\
& \geq-\lim _{n \rightarrow \infty} \frac{1}{n} \log \mathbb{P}\left(E_{4}^{c}\right)
\end{aligned}
$$

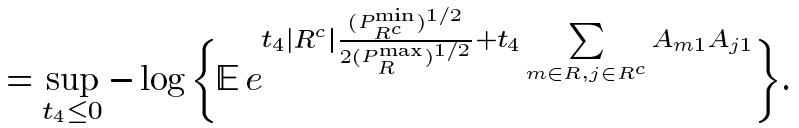

Recall the definition of $S_{R}$, which is $S_{R}=\sum_{m \in R} A_{m 1}$. Since $R^{c}$ and $R$ are by definition disjoint, and $\cosh (t) \leq e^{t^{2} / 2}$, it follows that

$$
\mathbb{E} e^{t_{4} S_{R^{c}} S_{R}}=\mathbb{E}\left(\cosh \left(t_{4} S_{R}\right)\right)^{\left|R^{c}\right|} \leq \mathbb{E} e^{\left|R^{c}\right| t_{4}^{2} S_{R}^{2} / 2} .
$$

Furthermore, by (46), this is further bounded from above by $\left(1-t_{4}^{2}|R|\left|R^{c}\right|\right)^{-1 / 2}$, as long as $t_{4}^{2}\left|R \| R^{c}\right| \leq 1$. Note that conditions $\left(\mathcal{P}_{1}\right)$ and $\left(\mathcal{P}_{4}\right)$ imply that $P_{R^{c}}^{\min }\left|R^{c}\right| \geq P^{\max }{ }_{R}|R|$ when $k$ is sufficiently large, because $P^{\max } R|R|$ is bounded and $\left|R^{c}\right|=$ $k(1-o(1))$. Substituting this yields

$$
\begin{aligned}
&-\lim _{n \rightarrow \infty} \frac{1}{n} \log \mathbb{P}\left(E_{1} \cap E_{2} \cap E_{3} \cap E_{4}^{c}\right) \\
& \geq \sup _{t_{4} \leq 0}-t_{4} \frac{\left|R^{c}\right|\left(P_{R^{c}}^{\min }\right)^{1 / 2}}{2\left(P^{\max }\right)^{1 / 2}}+\frac{1}{2} \log \left(1-t_{4}^{2}\left|R \| R^{c}\right|\right) \\
& \geq \sup _{t_{4} \leq 0}-t_{4} \frac{|R|^{1 / 2}\left|R^{c}\right|^{1 / 2}}{2}+\frac{1}{2} \log \left(1-t_{4}^{2}|R|\left|R^{c}\right|\right) .
\end{aligned}
$$

We substitute $t_{4}=-\frac{1}{2|R|^{1 / 2}\left|R^{c}\right|^{1 / 2}}$ to obtain a lower bound. Observe that the substituted $t_{4}$ indeed fulfills $t_{4}^{2}\left|R \| R^{c}\right| \leq 1$. Substitution of the above result yields

$$
\text { - } \lim _{n \rightarrow \infty} \frac{1}{n} \log \mathbb{P}\left(E_{1} \cap E_{2} \cap E_{3} \cap E_{4}^{c}\right) \geq \frac{1}{2} \log \frac{3}{4}+\frac{1}{4} .
$$

The second lemma is more involved than Lemma A.1.

Lemma A.2: For $|R|$ fixed and odd, under conditions $\left(\mathcal{P}_{1}-\mathcal{P}_{4}\right)$

- $\lim _{k \rightarrow \infty} \lim _{n \rightarrow \infty} \frac{1}{n} \log \mathbb{P}\left(E_{1} \cap E_{2} \cap E_{3}\right) \leq-\lim _{n \rightarrow \infty} \frac{1}{n} \log \mathbb{P}\left(E_{1}\right)$.

Proof: Cramér's theorem gives that

$$
\text { - } \lim _{n \rightarrow \infty} \frac{1}{n} \log \mathbb{P}\left(E_{1} \cap E_{2} \cap E_{3}\right)=\sup _{\underline{t} \in D}-\log \mathbb{E} e^{Y_{1}+Y_{2}+Y_{3}}
$$

where $\underline{t}=\left(t_{1}, t_{2}, t_{3}\right), D=\left\{\underline{t}: t_{1}, t_{3} \leq 0, t_{2} \geq 0\right\}$ and

$$
Y_{1}=t_{1}\left(\frac{|R|\left(P_{R}^{\max }\right)^{1 / 2}}{2\left(P_{R}^{\min }\right)^{1 / 2}}+\sum_{\substack{m, j \in R \\ m \neq j}} A_{m 1} A_{j 1}\right)
$$

$$
\begin{aligned}
Y_{2} & =t_{2}\left(\sum_{\substack{m, j \in R^{c} \\
m \neq j}} A_{m 1} A_{j 1}\right) \\
Y_{3} & =t_{3}\left(-|R|\left|R^{c}\right| \frac{\left(P_{R^{c}}^{\max } P_{R}^{\max }\right)^{1 / 2}}{2 P_{R^{c}}}+\sum_{m \in R, j \in R^{c}} A_{m 1} A_{j 1}\right) .
\end{aligned}
$$

We rewrite this, invoking $S_{A}=\sum_{j \in A} A_{j 1}$ as

$$
\begin{aligned}
& Y_{1}=t_{1}\left(|R|\left(\frac{\left(P^{\max } R\right)^{1 / 2}}{2\left(P_{R}^{\min }\right)^{1 / 2}}-1\right)+S_{R}^{2}\right) \\
& Y_{2}=t_{2}\left(-\left|R^{c}\right|+S_{R^{c}}^{2}\right) \\
& \left.Y_{3}=t_{3}\left(-|R|\left|R^{c}\right| \frac{\left(P^{\max } R^{c} P^{\max }\right.}{2 P_{R^{c}}}\right)^{1 / 2}+S_{R} S_{R^{c}}\right) .
\end{aligned}
$$

It is sufficient to prove that for $k \rightarrow \infty$, for all $t_{1}, t_{2}$

$$
\mathbb{E} e^{Y_{1}+Y_{2}+Y_{3}} \geq \mathbb{E} e^{Y_{1}}(1-o(1)) .
$$

Indeed, then

$$
\begin{aligned}
-\lim _{n \rightarrow \infty} \frac{1}{n} \log \mathbb{P}\left(E_{1}\right) & =\sup _{t_{1} \leq 0}-\log \mathbb{E} e^{Y_{1}} \\
& \leq \sup _{t_{1} \leq 0}\left\{-\log \mathbb{E} e^{Y_{1}+Y_{2}+Y_{3}}\right\}+o(1) \\
& \leq \sup _{\underline{t} \in D}\left\{-\log \mathbb{E} e^{Y_{1}+Y_{2}+Y_{3}}\right\}+o(1)
\end{aligned}
$$

so that for $k \rightarrow \infty$, the statement of the lemma follows. We abbreviate

$$
h\left(t_{1}, t_{2}, t_{3}\right)=\mathbb{E} e^{Y_{1}+Y_{2}+Y_{3}} .
$$

We will prove that

$$
h\left(t_{1}, t_{2}, t_{3}\right) \geq \mathbb{E} e^{Y_{1}}(1-o(1)), \quad k \rightarrow \infty .
$$

Instead of maximizing $-\log h\left(t_{1}, t_{2}, t_{3}\right)$, we will minimize $h\left(t_{1}, t_{2}, t_{3}\right)$. To do this, we will define an appropriate ellipse $\mathcal{E}$ with elements $t_{2}, t_{3}$ with $(0,0) \in \mathcal{E}^{0}$, the interior of $\mathcal{E}$. In order to show that the supremum of $-\log h\left(t_{1}, t_{2}, t_{3}\right)$ is attained in $\mathcal{E}^{0}$, it is sufficient to show that on the boundary of the ellipse $h\left(t_{1}, t_{2}, t_{3}\right)>\mathbb{E} e^{Y_{1}}$. Since $h\left(t_{1}, 0,0\right)=\mathbb{E} e^{Y_{1}}$ and $h$ is log-convex (since it is a moment-generating function), we can then conclude that $h\left(t_{1}, t_{2}, t_{3}\right)>\mathbb{E} e^{Y_{1}}$ outside the ellipse. Indeed, whenever $\left(t_{2}, t_{3}\right) \notin \mathcal{E}$, there exists a unique $0<\alpha<1$ such that $\alpha\left(t_{2}, t_{3}\right) \in \partial \mathcal{E}$. From convexity of $h$ and $h\left(t_{1}, \alpha\left(t_{2}, t_{3}\right)\right)>\mathbb{E} e^{Y_{1}}$ it follows that

$$
\begin{aligned}
\mathbb{E} e^{Y_{1}} & <h\left(\alpha\left(t_{1}, t_{2}\right), t_{3}\right)=h\left(\alpha\left(t_{1}, t_{2}\right)+(1-\alpha)(0,0), t_{3}\right) \\
& \leq \alpha h\left(t_{1}, t_{2}, t_{3}\right)+(1-\alpha) h\left(0,0, t_{3}\right) \\
& =\alpha h\left(t_{1}, t_{2}, t_{3}\right)+(1-\alpha) \mathbb{E} e^{Y_{1}} .
\end{aligned}
$$

It immediately follows that $h\left(t_{1}, t_{2}, t_{3}\right)>\mathbb{E} e^{Y_{1}}$, so that $\left(t_{1}, t_{2}, t_{3}\right)$ cannot be the minimizer of $h\left(t_{1}, t_{2}, t_{3}\right)$ over $D$. When $\left(t_{2}, t_{3}\right) \in \mathcal{E}^{0}$, we can prove

$$
h\left(t_{1}, t_{2}, t_{3}\right) \geq \mathbb{E} e^{Y_{1}}(1-o(1)), \quad \text { for } k \rightarrow \infty .
$$

Since $e^{x} \geq 1+x+x^{2} / 2+x^{3} / 6$, we have $\mathbb{E} e^{Y_{1}+Y_{2}+Y_{3}}$ bounded from below by

$$
\mathbb{E}\left(e^{Y_{1}}\left(1+Y_{2}+Y_{3}+\frac{1}{2}\left(Y_{2}+Y_{3}\right)^{2}+\frac{1}{6}\left(Y_{2}+Y_{3}\right)^{3}\right)\right) .
$$


We will first calculate the required moments. Since $|R|$ is odd, we have that $S_{R}^{2} \geq 1$, so that

$$
\begin{aligned}
\mathbb{E} e^{Y_{1}} Y_{2} & =0 \\
\mathbb{E} e^{Y_{1}} Y_{2} Y_{3} & =0 \\
\mathbb{E} e^{Y_{1}} Y_{3} & =\mathbb{E} e^{Y_{1}}\left(-t_{3}\left|R \| R^{c}\right| \frac{\left(P_{R^{c}}^{\max } P_{R}^{\max }\right)^{1 / 2}}{2 P_{R^{c}}}\right) \\
\mathbb{E} e^{Y_{1}} Y_{2}^{2} & =\mathbb{E} e^{Y_{1}} t_{2}^{2}\left(3\left|R^{c}\right|^{2}+2\left|R^{c}\right|\right) \geq \mathbb{E} e^{Y_{3}} 3 t_{2}^{2}\left|R^{c}\right|^{2} \\
\mathbb{E} e^{Y_{1}} Y_{3}^{2} & =\mathbb{E} e^{Y_{1}}\left(t_{3}^{2} \frac{|R|^{2}\left|R^{c}\right|^{2} P_{R^{c}}^{\max } P_{R}^{\max }}{4 P_{R^{c}}^{2}}+t_{3}^{2} S_{R}^{2}\left|R^{c}\right|\right) \\
& \geq \mathbb{E} e^{Y_{1}} t_{3}^{2}\left|R^{c}\right| .
\end{aligned}
$$

Concerning $\mathbb{E} e^{Y_{1}}\left(Y_{2}+Y_{3}\right)^{3}$

$$
\mathbb{E} e^{Y_{1}}\left(Y_{2}+Y_{3}\right)^{3} \geq-4 \mathbb{E} e^{Y_{1}}\left(\left|Y_{2}^{3}\right|+\left|Y_{3}^{3}\right|\right)
$$

Using (91) and $\left|S_{R}\right| \leq|R|$ results in

$$
\left|Y_{3}^{3}\right| \leq 4\left|t_{3}\right|^{3}\left(|R|^{3}\left|R^{c}\right|^{3} \frac{\left(P_{R^{c}}^{\max }\right)^{3 / 2}\left(P_{R}^{\max }\right)^{3 / 2}}{8 P_{R^{c}}^{3}}+|R|^{3}\left|S_{R^{c}}^{3}\right|\right)
$$

and

$$
\left|Y_{2}^{3}\right| \leq 4 t_{2}^{3}\left(\left|R^{c}\right|^{3}+\left|S_{R^{c}}^{6}\right|\right)
$$

so that (using $\mathbb{E}\left|S_{R^{c}}^{m}\right| \leq C\left|R^{c}\right|^{m / 2}$ for some $C<\infty$ )

$$
\begin{aligned}
&-\mathbb{E} e^{Y_{1}}\left(\left|Y_{2}^{3}\right|+\left|Y_{3}^{3}\right|\right) \\
& \geq-\mathbb{E} e^{Y_{1}}\left(C\left|t_{3}\right|^{3}|R|^{3}\left|R^{c}\right|^{3} \frac{\left(P_{R^{c}}^{\max }\right)^{3 / 2}\left(P_{R}^{\max }\right)^{3 / 2}}{P_{R^{c}}^{3}}\right. \\
&\left.\quad+C\left|t_{3}\right|^{3}|R|^{3}\left|R^{c}\right|^{3 / 2}+C t_{2}^{3}\left|R^{c}\right|^{3}\right) .
\end{aligned}
$$

We next use the moments of (93) to obtain

$$
\begin{array}{r}
h\left(t_{1}, t_{2}, t_{3}\right) \\
\geq \mathbb{E} e^{Y_{1}}\left(1+2\left|R^{c}\right|^{2} t_{2}^{2}-t_{3}|R|\left|R^{c}\right| \frac{\left(P_{R^{c}}^{\max } P^{\max }{ }_{R}\right)^{1 / 2}}{2 P_{R^{c}}}\right. \\
\left.\quad+\frac{t_{3}^{2}\left|R^{c}\right|}{2}+e\left(t_{2}, t_{3}\right)\right) \\
=\mathbb{E} e^{Y_{1}}\left(1-\mathcal{H}+\frac{3\left|R^{c}\right|^{2}}{2} t_{2}^{2}+\frac{\left|R^{c}\right|}{2}\left(t_{3}-t_{3}^{*}\right)^{2}+e\left(t_{2}, t_{3}\right)\right)
\end{array}
$$

where

$$
\begin{aligned}
\mathcal{H}= & \frac{|R|^{2}\left|R^{c}\right| P_{R^{c}}^{\max } P_{R}^{\max }}{8 P_{R^{c}}^{2}} \\
t_{3}^{*}= & -\frac{|R|\left(P_{R^{c}}^{\max } P_{R}^{\max }\right)^{1 / 2}}{2 P_{R^{c}}} \\
e\left(t_{1}, t_{2}\right)= & -C t_{2}^{3}\left|R^{c}\right|^{3}-C\left|t_{1}\right|^{3}|R|^{3}\left|R^{c}\right|^{3 / 2} \\
& -C\left|t_{1}\right|^{3}|R|^{3}\left|R^{c}\right|^{3} \frac{\left(P_{R^{c}}^{\max }\right)^{3 / 2}\left(P^{\max }{ }_{R}\right)^{3 / 2}}{P_{R^{c}}^{3}} .
\end{aligned}
$$

According to $\left(\mathcal{P}_{1}\right)$, we have that $P_{R} \leq P_{R}^{\max }|R| \leq \delta^{-1}|R|$ is bounded, so that $P=P_{R^{c}}+P_{R}=P_{R^{c}}(1-o(1))$. This implies, using $\left(\mathcal{P}_{1}-\mathcal{P}_{4}\right)$, that as $k \rightarrow \infty$

$$
\mathcal{H} \leq \frac{|R|^{2} k P^{\max } \delta^{-1}}{8 P^{2}(1-o(1))}=\frac{|R|^{2} \delta^{-1}}{8} \frac{P^{\max }}{k} \frac{k^{2}}{P^{2}}(1+o(1)) \rightarrow 0 .
$$

We define the ellipse $\mathcal{E}$ as

$$
\mathcal{E}=\left\{\left(t_{2}, t_{3}\right): \frac{3\left|R^{c}\right|^{2}}{2} t_{2}^{2}+\frac{\left|R^{c}\right|}{2}\left(t_{3}-t_{3}^{*}\right)^{2} \leq 2 \mathcal{H}\right\} .
$$

To show that on $\partial \mathcal{E}, h\left(t_{1}, t_{2}, t_{3}\right)>\mathbb{E} e^{Y_{1}}$, it is sufficient to show that for every $|R|$ fixed

$$
\left|e\left(t_{2}, t_{3}\right)\right|=o(\mathcal{H})
$$

Indeed, on $\partial \mathcal{E}$, according to (95)

$$
h\left(t_{1}, t_{2}, t_{3}\right)=\mathbb{E} e^{Y_{1}}\left(1-\mathcal{H}+2 \mathcal{H}+e\left(t_{2}, t_{3}\right)\right)>\mathbb{E} e^{Y_{1}} .
$$

Therefore, we focus on $e\left(t_{2}, t_{3}\right)$ for $\left(t_{2}, t_{3}\right) \in \partial \mathcal{E}$. Note that for $\left(t_{2}, t_{3}\right) \in \mathcal{E}$ by the triangle inequality

$$
\begin{aligned}
& \left|t_{2}\right| \leq \frac{1}{\sqrt{6}} \frac{|R|\left(P_{R^{c}}^{\max } P^{\max }{ }_{R}\right)^{1 / 2}}{\left|R^{c}\right|^{1 / 2} P_{R^{c}}} \\
& \left|t_{3}\right| \leq \frac{|R|\left(P_{R^{c}}^{\max } P_{R}^{\max }\right)^{1 / 2}}{P_{R^{c}}} .
\end{aligned}
$$

Substituting this into the formula for $e\left(t_{2}, t_{3}\right)$ gives

$$
\begin{aligned}
\left|e\left(t_{2}, t_{3}\right)\right| \leq & C \frac{|R|^{3}\left(P_{R^{c}}^{\max } P_{R}^{\max }\right)^{3 / 2}}{P_{R^{c}}^{3}} \\
& \times\left(|R|^{3}\left|R^{c}\right|^{3} \frac{\left(P_{R^{c}}^{\max } P_{R}^{\max }\right)^{3 / 2}}{P_{R^{c}}^{3}}+|R|^{3}\left|R^{c}\right|^{3 / 2}\right) \\
& +C \frac{|R|^{3}\left(P_{R^{c}}^{\max } P_{R}^{\max }\right)^{3 / 2}}{\left|R^{c}\right|^{3 / 2} P_{R^{c}}}\left|R^{c}\right|^{3} \\
\leq & C\left(\mathcal{H}^{3}+|R|^{3} \mathcal{H}^{3 / 2}\right)+C \mathcal{H}^{3 / 2} .
\end{aligned}
$$

Since $\mathcal{H}=o(1)$ according to (95) and $|R|$ is fixed, indeed $\left|e\left(t_{2}, t_{3}\right)\right|=o(\mathcal{H})$. This proves that

$$
h\left(t_{1}, t_{2}, t_{3}\right)>\mathbb{E} e^{Y_{1}}, \quad\left(t_{2}, t_{3}\right) \notin \mathcal{E} .
$$

Finally, for $\left(t_{2}, t_{3}\right) \in \mathcal{E}^{0}$, we use the preceding results to obtain $h\left(t_{1}, t_{2}, t_{3}\right) \geq \mathbb{E} e^{Y_{1}}\left(1-\mathcal{H}+\frac{3\left|R^{c}\right|^{2}}{2} t_{2}^{2}+\frac{\left|R^{c}\right|}{2}\left(t_{3}-t_{3}^{*}\right)^{2}-o(\mathcal{H})\right)$.

Minimization over $t_{2}$ and $t_{3}$ of the right-hand side clearly gives $t_{2}=0$ and $t_{3}=t_{3}^{*}$, so that for all $\left(t_{2}, t_{3}\right) \in \mathcal{E}$

$$
h\left(t_{1}, t_{2}, t_{3}\right) \geq \mathbb{E} e^{Y_{1}}(1-\mathcal{H}-o(\mathcal{H})) .
$$

Finally, since $\mathcal{H}=o(1), 1-\mathcal{H}-o(\mathcal{H})=1-o(1)$. Together with (99), this proves the claim in (92).

Lemma A.3: For $|R| \rightarrow \infty$, under condition $\left(\mathcal{P}_{1}\right)$

$$
-\lim _{n \rightarrow \infty} \frac{1}{n} \log \mathbb{P}\left(E_{1}\right) \leq \frac{1}{2} \log 2-\frac{1}{4}+o(1) .
$$


Proof: Clearly

$$
-\lim _{n \rightarrow \infty} \frac{1}{n} \log \mathbb{P}\left(E_{1}\right)=\sup _{t_{1} \leq 0}\left\{-\log \mathbb{E} e^{Y_{1}}\right\}
$$

where

$$
Y_{1}=t_{1}\left(|R|\left(\frac{\left(P_{R}^{\max }\right)^{1 / 2}}{2\left(P_{R}^{\min }\right)^{1 / 2}}-1\right)+S_{R}^{2}\right)
$$

When we prove

$$
\lim _{|R| \rightarrow \infty} \sup _{t_{1} \leq 0}\left\{-\log \mathbb{E} e^{Y_{1}}\right\} \leq \frac{1}{2} \log 2-\frac{1}{4}
$$

we conclude that for $|R|$ sufficiently large

$$
-\lim _{n \rightarrow \infty} \frac{1}{n} \log \mathbb{P}\left(E_{1}\right) \leq \frac{1}{2} \log 2-\frac{1}{4}+o(1) .
$$

Thus, it remains to show (100). We denote

$$
j_{R}=\frac{1}{2} \frac{\left(P_{R}^{\max }\right)^{1 / 2}}{\left(P_{R}^{\min }\right)^{1 / 2}}-\frac{1}{2} .
$$

The crucial step in this part of the proof is that we are still allowed to choose $R$. So far, we have only assumed that $R \subset$ $\left\{j: P_{j} \in[\delta, 1 / \delta]\right\}$ and that $|R|$ is odd. By condition $\left(\mathcal{P}_{1}\right)$, we can choose $R$ such that whenever $|R|=r$, there exists a $\bar{P}$ with $\max _{m \in R}\left|P_{m}-\bar{P}\right| \leq \bar{P} / r$. This implies $P_{R}^{\max } \leq(1+1 /|R|) \bar{P}$ and $P_{R}^{\min } \geq(1-1 /|R|) \bar{P}$, so that for $|R| \rightarrow \infty$

$$
0 \leq j_{R} \leq \frac{1}{2} \sqrt{\frac{1+\frac{1}{|R|}}{\left(1-\frac{1}{|R|}\right)}}-\frac{1}{2}=\sqrt{1+\frac{2}{|R|-1}}-1 \rightarrow 0 .
$$

We next write

$$
\begin{aligned}
-\inf _{t_{1} \leq 0} \log \mathbb{E} & \exp \left(t_{1}\left(\frac{P_{R}^{\max }}{2 P_{R}^{\min }}-\frac{1}{2}\right)+t_{1} S_{R}^{2}\right) \\
& =-\inf _{t_{1} \leq 0} \log \mathbb{E} \exp \left(t_{1} j_{R}+t_{1}\left(S_{R}^{2}-\frac{|R|}{2}\right)\right) .
\end{aligned}
$$

We can now follow the argument in the proof of Lemma V.4, together with the observation that whenever $X_{n} \stackrel{\mathcal{D}}{\rightarrow} X$ and $c_{n} \rightarrow c$ that also $c_{n} X_{n} \stackrel{\mathcal{D}}{\rightarrow} c X$. Indeed, in all weak convergence arguments $-1 /|R| \leq t_{1} \leq 0$, so that we are allowed to replace $e^{t_{1} j_{R}}$ by its limit 1 . This proves that indeed for $|R|$ large

$$
-\inf _{t_{1} \leq 0} \log \mathbb{E} e^{Y_{1}} \leq \frac{1}{2} \log 2-\frac{1}{4}+o(1) .
$$

This completes the proof of Theorem III.2 b) in the case of unequal powers.

\section{ACKNOWLEDGMENT}

We thank the anonymous referees for many useful comments.

\section{REFERENCES}

[1] R. M. Buehrer and B. D. Woerner, "Analysis of adaptive multistage interference cancellation for CDMA using an improved Gaussian approximation," IEEE Trans. Commun., vol. 44, pp. 1308-1329, Oct. 1996.

[2] R. M. Buehrer, S. P. Nicoloso, and S. Gollamudi, "Linear versus nonlinear interference cancellation," J. Commun. and Networks, vol. 1, no. 2, 1999.

[3] A. C. C. Coolen, "Dynamics of recall and association-A compact review," in The Handbook of Brain Theory and Neural Networks, M. A. Arbib, Ed. Cambridge, MA: MIT Press, 2002.

[4] P. Floréen and P. Orponen, "On the computational complexity of analyzing Hopfield nets," Complex Syst., vol. 3, no. 6, pp. 577-587, 1989.

[5] A. Dembo and O. Zeitouni, Large Deviations Techniques and Applications, 2nd ed. New York: Springer-Verlag.

[6] R. Gold, "Optimal binary sequences for spread spectrum multiplexing," IEEE Trans. Inform. Theory, vol. IT-13, pp. 619-621, Oct. 1967.

[7] G. R.+ Grimmett and D. R. Stirzaker, Probability and Random Processes, 2nd ed. Oxford, U.K.: Oxford Univ. Press, 1992.

[8] R. van der Hofstad, G. Hooghiemstra, and M. J. Klok, "Large deviations for code division multiple access systems," SIAM J. Appl. Math, vol. 62, no. 3, pp. 1044-1064, 1999.

[9] R. van der Hofstad and M. J. Klok, "Improving the performance of third generation wireless communication systems," preprint, 2001.

[10] F. den Hollander, Large Deviations, ser. Fields Institute Monographs. Providence, RI: Amer. Math. Soc., 2000.

[11] M. Juntti, "Multiuser demodulation for DS-CDMA systems in fading channels," Ph.D. dissertation, Univ. Oulu, Oulu, Finland, 1998.

[12] T. Kasami, "Weight distribution formula for some class of cyclic codes," Coordinated Science Lab., Univ. Illinois, Urbana, Tech. Rep. R-285, 1966.

[13] M. J. Klok, "On the asymptotic rate of error probabilities in third generation wireless systems," preprint, 2001.

[14] — "Importance sampling for DS-CDMA sytemes with 1-stage HD-PIC," in Proc. WPMC'01, Aalborg, Denmark, Sept. 2001.

[15] - "Performance analysis of advanced third generation receivers," Ph.D. dissertation, Delft Univ. Technol., Delft, The Netherlands, 2001.

[16] M. Latva-aho, "Advanced receivers for wideband CDMA systems," Ph.D. dissertation, Univ. Oulu, Oulu, Finland, 1999.

[17] S. Moshavi, "Multi-user detection for DS-CDMA communications," IEEE Commun. Mag., pp. 124-136, Oct. 1996.

[18] R. Prasad, W. Mohr, and W. Konhäuser, Third Generation Mobile Communication Systems. Norwood, MA: Artech House, 2000.

[19] J. S. Sadowski and R. K. Bahr, "Direct-sequence spread-spectrum multiple-access communications with random signature sequences: A large deviations analysis," IEEE Trans. Inform. Theory, vol. 37, pp. 514-527, May 1991.

[20] M. K. Varanasi and B. Aazhang, "Multistage detection in asynchronous code-division multiple acces communications," IEEE Trans. Commun., vol. 38, pp. 509-519, Apr. 1990

[21] S. Verdú, "Minimum probability of error for asynchronous Gaussian multiple access," IEEE Trans. Inform. Theory, vol. IT-32, pp. 85-96, Jan. 1986.

[22] G. Xue, J. Weng, T. Le-Ngoc, and S. Tahar, "An analytical model for performance evaluation of multistage parallel interference cancellation detectors in CDMA systems," in Proc. Vehicular Technology Conf. (VTC'99) Fall, Amsterdam, The Netherlands, 1999, pp. 1144-1149. 